\title{
Effects of stencil width on surface ocean geostrophic velocity and vorticity estimation from gridded satellite altimeter data
}

\author{
Brian K. Arbic, ${ }^{1}$ Robert B. Scott, ${ }^{2,3}$ Dudley B. Chelton, ${ }^{4}$ James G. Richman, ${ }^{5}$ \\ and Jay F. Shriver ${ }^{5}$ \\ Received 7 June 2011; revised 20 January 2012; accepted 23 January 2012; published 17 March 2012.
}

[1] This paper examines the effect of "stencil width" on surface ocean geostrophic velocity and vorticity estimated from differentiating gridded satellite altimeter sea surface height products. In oceanographic applications, the value of the first derivative at a central grid point is generally obtained by differencing the sea surface heights at adjacent grid points. This is called a "three-point stencil centered difference". Here the stencil width is increased from three to five, seven, and nine points, using well-known formulae from the numerical analysis literature. The discrepancies between velocities computed with successive stencils decreases with increasing stencil width, suggesting that wide stencil results are more reliable. Significant speed-dependent biases (up to 10-20\%) are found between results computed from three-point stencils versus those computed from wider stencils. The geostrophic velocity, and the variance of geostrophic velocity, are underestimated with thin stencils. Similar results are seen in geostrophic velocities computed from high-resolution model output. In contrast to the case when three-point stencils are used, wider stencils yield estimates of the anisotropy of velocity variance that are insensitive to the differences in grid spacing between two widely used altimeter products. Three-point stencils yield incorrect anisotropies on the $1 / 4^{\circ}$ anisotropic AVISO grid; we recommend the use of 7-point stencils. Despite the demonstrated inadequacies of the three-point stencils, the conclusions of earlier studies based on them, that the zonally averaged midlatitude eddy kinetic energy field is nearly isotropic, are found to pertain also with wider stencils. Finally, the paper also examines the strengths and limitations of applying noise-suppressing differentiators, versus classic centered differences, to altimeter data.

Citation: Arbic, B. K., R. B. Scott, D. B. Chelton, J. G. Richman, and J. F. Shriver (2012), Effects of stencil width on surface ocean geostrophic velocity and vorticity estimation from gridded satellite altimeter data, J. Geophys. Res., 117, C03029, doi:10.1029/2011JC007367.

\section{Introduction}

[2] Satellite altimeter measurements of sea surface height [Fu and Cazenave, 2001] have revolutionized physical oceanography, enabling and enhancing numerous avenues of study. To name just three of many applications, satellite altimetry data has been utilized to estimate sea surface height and kinetic energy spectra [Stammer, 1997; Xu and Fu, 2011], reveal the inverse kinetic energy cascade of surface

\footnotetext{
${ }^{1}$ Department of Earth and Environmental Sciences, University of Michigan, Ann Arbor, Michigan, USA.

${ }^{2}$ Institute for Geophysics, Jackson School of Geosciences, University of Texas at Austin, Austin, Texas, USA.

${ }^{3}$ Département de Physique and LPO, Université de Bretagne Occidental, Brest, France.

${ }^{4}$ College of Oceanic and Atmospheric Sciences, Oregon State University, Corvallis, Oregon, USA.

${ }^{5}$ Oceanography Division, Naval Research Laboratory, Stennis Space Center, Mississippi, USA.

Copyright 2012 by the American Geophysical Union. 0148-0227/12/2011JC007367
}

oceanic flows [Scott and Wang, 2005], and document the propagation paths of mesoscale eddies [Chelton et al., 2007, 2011]. A recent brief review of applications of altimeter data is given by Scott et al. [2010].

[3] Two important products of satellite altimeter data are the ocean surface velocity and vorticity fields, extracted via the geostrophic relations. Geostrophic velocities are obtained from first derivatives of the sea surface height $\eta$ :

$$
\begin{gathered}
u=-\frac{g}{f} \frac{\partial \eta}{\partial y} \\
v=\frac{g}{f} \frac{\partial \eta}{\partial x}
\end{gathered}
$$

where $u$ is zonal velocity (velocity in the east-west direction, with eastward velocities being positive), $v$ is meridional velocity (velocity in the north-south direction, with northward velocities being positive), $g$ is gravitational acceleration, $f$ is the Coriolis parameter, $x$ is the zonal spatial 
coordinate, and $y$ is the meridional spatial coordinate. Geostrophic vorticity $\zeta$ is obtained from first derivatives of the velocity field, and therefore second derivatives of sea surface height:

$$
\zeta=\frac{\partial v}{\partial x}-\frac{\partial u}{\partial y}=\frac{g}{f}\left(\frac{\partial^{2} \eta}{\partial x^{2}}+\frac{\partial^{2} \eta}{\partial y^{2}}\right)-\frac{g \beta}{f^{2}} \frac{\partial \eta}{\partial y},
$$

where $\beta=\partial f / \partial y$ is the planetary vorticity gradient. The ratio of the last term on the right-hand side in (3) to the second derivative terms scales as $\beta L / f$, where $L$ is a typical horizontal length scale. Under the traditional "beta plane approximation", this ratio is small in midlatitudes [e.g., Pedlosky, 1987; Vallis, 2006].

[4] Here we examine the effects of "stencil width" on the estimation of geostrophic velocities and vorticities from gridded altimeter sea surface height products. By "stencil width" we mean the number of grid points utilized to estimate the finite difference approximation to the derivative on a grid. As we will discuss, varying the stencil width is another way of varying the accuracy of the derivative estimate. Our focus will be on the velocity fields, but we will include a brief discussion of the vorticity field. In the vast majority of oceanographic applications, geostrophic velocities are estimated from a "three-point stencil centered difference", in which sea surface height values at adjacent grid points are differenced to determine velocities at a central grid point. However, inspection of the substantial literature on numerical methods [e.g., Strikwerda, 2004; Mathews and Fink, 2004] reveals well-known formulae for computing first derivatives of fields via centered differences taken with wider stencils, for instance, 5-point, 7-point, and 9-point stencils. (We also found the following websites helpful: http:// www.holoborodko.com/pavel/numerical_methods/numericalderivative/, which was especially helpful and is hereinafter referred to as Holoborodko (2011); http://en.wikipedia. org/wiki/Five-point_stencil; http://reference.wolfram.com/ mathematica/tutorial/NDSolvePDE.html\#c:4.) It is of interest to determine whether usage of wider stencils would alter the velocity and vorticity estimates. As we shall see from the numerical analysis literature, derivatives estimated with wider stencils feature reduced errors over those computed with 3-point stencils.

[5] Since the errors in derivative estimates are a function of grid spacing, it is also of interest to determine how stencil width affects velocity estimates computed on grids of differing grid spacings. For example, we will examine derivatives computed on the widely-used isotropic $1 / 3^{\circ}$ Mercator grid reference product distributed by the Archiving, Validation, and Interpretation of Satellite Oceanographic (AVISO) gridded altimeter data [Le Traon et al., 1998; Ducet et al., 2000], versus derivatives computed on the (also widely used) anisotropic $1 / 4^{\circ}$ latitude-longitude grid reference product put out by AVISO. By "isotropic grid", we mean a grid in which the spacing in kilometers is the same in the east-west and north-south directions. This is the case for the $1 / 3^{\circ}$ Mercator AVISO grid, in which the zonal grid spacing is fixed in degrees of longitude and the meridional grid spacing decreases in degrees of latitude with the cosine of latitude so as to match the zonal grid spacing in kilometers. On an "anisotropic grid", such as the $1 / 4^{\circ}$ AVISO latitudelongitude grid, the grid spacing in the north-south direction is the same across the entire grid, while grid spacing in the east-west direction is fixed in degrees of longitude but decreases in kilometers with the cosine of latitude. Since the $1 / 4^{\circ}$ product is simply a bi-linear interpolation of the $1 / 3^{\circ}$ product, we might expect that derivatives calculated on the two grids should yield very similar results. We will show that this is the case only if wide stencils are utilized. Since the derivative in the zonal direction yields meridional velocity while the derivative in the meridional direction yields zonal velocity, this discussion will be useful for the estimation of anisotropy in the oceanic kinetic energy field [Ducet et al., 2000; Scott et al., 2008; Scharffenberg and Stammer, 2010].

[6] We will also test whether the behaviors seen when differentiating sea surface heights in altimetric data sets are seen as well when differentiating sea surface heights in highresolution numerical models. Models and altimetric data sets exhibit different types of errors, hence a consistency in results obtained from the two sources would demonstrate that the impact of stencil width is not simply an artifact of the particular nature of gridded altimeter products. Here we utilize results from NLOM, the Naval Research Laboratory Layered Ocean Model [Hurlburt and Thompson, 1980; Wallcraft et al., 2003]. NLOM is run as a data-assimilative nowcast/forecast model by the United States Navy [Shriver et al., 2007]. Here for simplicity we utilize a snapshot from a forward (non-assimilative) run of NLOM. Since the model is on a much higher horizontal resolution grid than is the altimeter data, we can use either subsampled or smoothed versions of the model to further test the effect of stencil widths for differing horizontal grid resolutions.

[7] Most of the paper is about "classic" centered differences, which operate well on noiseless data or on numerical models. Later in the paper we will examine the impact of data noise on velocity estimates. We will also discuss application of noise-suppressing differentiators, which have been widely discussed in the chemical and signal processing literature [Savitzky and Golay, 1964; Steiner et al., 1972; Gorry, 1990; Luo et al., 2005; Holoborodko, 2011], to altimeter data.

[8] This paper is organized as follows. In section 2, we list the formulae for first and second derivatives computed as 3-, 5-, 7-, and 9-point stencil "classic" centered differences, taken from standard references in the numerical finite difference literature. We also list the leading order error terms associated with the derivative estimates. In section 3, we show the results of a "von Neumann analysis" [LeVeque, 2007], which demonstrates that the deviations of classic centered-difference estimates of derivatives from estimates made by an "ideal differentiator" (to be explained in section 3) decrease as stencil width increases. We utilize results from an idealized quasi-geostrophic turbulence model in this exercise. Since these deviations depend on length scale, we include and discuss a wave number spectrum of the AVISO data in section 3. We then show, in section 4, that the differences between geostrophic velocities computed with successively wider stencils decreases with increasing stencil width. Section 4 utilizes fields of sea surface height taken from the weekly $1 / 3^{\circ}$ and $1 / 4^{\circ}$ AVISO products as well as from NLOM. Section 5 presents a brief discussion of the alterations to the classic centered difference formulae when non-uniform grid spacing is taken into account. This is a small but measurable effect, relevant for zonal velocities computed on the $1 / 3^{\circ}$ AVISO grid. In section 6 , we present a 
Table 1. Formulae for Classic Centered Differences

\begin{tabular}{|c|c|c|}
\hline$N^{\mathrm{a}}$ & Formulae & $E^{\mathrm{b}}$ \\
\hline \multicolumn{3}{|c|}{ First Derivative ${ }^{\mathrm{c}}$} \\
\hline 3 & $\frac{\eta(r+h)-\eta(r-h)}{2 h}$ & $\frac{h^{2} \eta^{(3)}(r)}{6}$ \\
\hline 5 & $-\eta(r+2 h)+8 \eta(r+h)-8 \eta(r-h)+\eta(r-2 h)$ & $-\frac{h^{4} \eta^{(5)}(r)}{2}$ \\
\hline & $\begin{array}{c}12 \mathrm{~h} \\
\end{array}$ & 30 \\
\hline 7 & $\frac{\eta(r+3 h)-9 \eta(r+2 h)+45 \eta(r+h)-45 \eta(r-h)+9 \eta(r-2 h)-\eta(r-3 h)}{60 h}$ & $\frac{h^{6} \eta^{(7)}(r)}{140}$ \\
\hline 9 & $\frac{-3 \eta(r+4 h)+32 \eta(r+3 h)-168 \eta(r+2 h)+672 \eta(r+h)-672 \eta(r-h)+168 \eta(r-2 h)-32 \eta(r-3 h)+3 \eta(r-4 h)}{840 h}$ & $-\frac{h^{8} \eta^{(9)}(r)}{630}$ \\
\hline \multicolumn{3}{|c|}{ Second Derivative ${ }^{\mathrm{d}}$} \\
\hline 3 & $\frac{\eta(r+n)-2 \eta(r)+\eta(r-n)}{h^{2}}$ & $\frac{n+1}{12}$ \\
\hline 5 & $\frac{-\eta(r+2 h)+16 \eta(r+h)-30 \eta(r)+16 \eta(r-h)-\eta(r-2 h)}{12 h^{2}}$ & $-\frac{h^{4} \eta^{(6)}(r)}{90}$ \\
\hline 7 & $\frac{2 \eta(r+3 h)-27 \eta(r+2 h)+270 \eta(r+h)-490 \eta(r)+270 \eta(r-h)-27 \eta(r-2 h)+2 \eta(r-3 h)}{180 h^{2}}$ & $\frac{h^{6} \eta^{(8)}(r)}{560}$ \\
\hline 9 & $\frac{-9 \eta(r+4 h)+128 \eta(r+3 h)-1008 \eta(r+2 h)+8064 \eta(r+h)-14350 \eta(r)+8064 \eta(r-h)-1008 \eta(r-2 h)+128 \eta(r-3 h)-9 \eta(r-4 h)}{5040 h^{2}}$ & $-\frac{h^{8} \eta^{(10)}(r)}{3150}$ \\
\hline
\end{tabular}

brief discussion of geostrophic vorticity computed from AVISO data with classic centered differences. This discussion continues to demonstrate the advantages of wider stencils. In section 7, we present a stochastic model which qualitatively explains some of the "speed-dependent biases" seen in the three-point stencil results of section 4 . In section 8 , we show that consistent estimates of the anistropy of kinetic energy variance on anisotropically versus isotropically gridded altimeter products can be obtained if and only if wide stencils are utilized. Section 9 examines the impact of noise on the derivative estimates, and introduces some noisesuppressing differentiators taken from Holoborodko (2011). We argue that for current-generation altimeter data (AVISO), the merits of suppressing noise at small scales are outweighed by the need to retain near "ideal differentiation" at larger scales, which the wider stencil classic centered differences provide. In section 10 we present a brief summary and discussion of our results.

\section{Formulae for N-Point Stencil Classic Centered Differences}

[9] For geostrophic velocity and vorticity estimation, we need to compute partial first and second derivatives of the sea surface height $\eta$ with respect to the east-west coordinate $x$ and north-south coordinate $y$. Let $r$ be a generic coordinate, representing either $x$ or $y$. Suppose further that we take the grid spacing of an altimeter product to be $h$. For the moment we assume uniform grid spacing. The 3-, 5-, 7-, and 9-point stencil estimates of the first derivative utilize 1, 2, 3, and 4 grid points, respectively, on either side of the grid point at which the derivative is required. For instance, the 3-point stencil utilizes the value of $\eta$ at $r+h$ and $r$ - $h$ to estimate the first derivative at $r$, the 5-point stencil utilizes the value of $\eta$ at $r+2 h, r+h, r-h$, and $r-2 h$ to estimate this derivative, and so on. Table 1 gives the formulae for the first and second partial derivatives of $\eta$ computed as classic centered differences on the 3-, 5-, 7-, and 9-point stencils, as well as the leading-order error term $E$ in the estimate [e.g., Strikwerda, 2004; Mathews and Fink, 2004] (http://www.holoborodko. com/pavel/numerical_methods/numerical-derivative/, http://en. wikipedia.org/wiki/Five-point_stencil, http://reference.wolfram.
com/mathematica/tutorial/NDSolvePDE.html\#c:4). Note that superscripts in parentheses denote order of derivative. Thus, for example, $\eta^{(9)}(r)$ denotes the ninth derivative of $\eta(r)$. Note also that the denominator in the error term increases with stencil width. Therefore we expect the differences in estimates made with successive stencils to decrease as the stencil widens.

[10] For the benefit of readers not familiar with the numerical finite difference methods literature, the derivation of the first derivative and error formulae is sketched out below. (For more detail, the reader can consult the numerical analysis references listed above, or Cushman-Roisin and Beckers [2010, chapter 1].) Using the 9-point stencil computation as an example, we write the derivative of sea surface height at a given grid point as a linear combination of sea surface heights at the four nearest grid points on each side,

$$
\frac{\partial \eta}{\partial r} \approx \frac{1}{h} \sum_{n} c_{n} \eta(r+n h), \quad n=-4,-3,-2,-1,1,2,3,4 .
$$

For each value of $n$, we write a Taylor series

$$
\begin{aligned}
\eta(r+n h) \approx & \eta(r)+(n h) \eta^{(1)}(r)+\frac{(n h)^{2} \eta^{(2)}(r)}{2 !} \\
& +\frac{(n h)^{3} \eta^{(3)}(r)}{3 !} \ldots+\frac{(n h)^{9} \eta^{(9)}(r)}{9 !}
\end{aligned}
$$

where we truncate the series at the ninth-derivative term. Inserting the Taylor series into (4) yields

$$
\begin{aligned}
\frac{\partial \eta}{\partial r} \approx & \frac{F_{0}\left(c_{n}\right) \eta(r)}{h}+F_{1}\left(c_{n}\right) \eta^{(1)}(r)+F_{2}\left(c_{n}\right) h \eta^{(2)}(r)+\ldots \\
& +F_{9}\left(c_{n}\right) h^{8} \eta^{(9)}(r)
\end{aligned}
$$

where $F_{0}\left(c_{n}\right), F_{1}\left(c_{n}\right), \ldots F_{9}\left(c_{n}\right)$ are simple linear combinations of the coefficients $c_{n}$. In order that only the first derivative is retained in the right-hand side of (6), we want $F_{1}\left(c_{n}\right)$ to be unity, and $F_{n}$ for $n \neq 1$ to be zero for as many $n$ as possible. For a 9-point stencil we are able to set the $F_{n}$ to zero for $n \neq 1$, all the way up to $F_{8}$. Setting $F_{0}\left(c_{n}\right)=F_{2}\left(c_{n}\right)=$ $F_{4}\left(c_{n}\right)=F_{6}\left(c_{n}\right)=0$ yields $c_{-n}=-c_{n}$ for $n=1,2,3,4$. This 

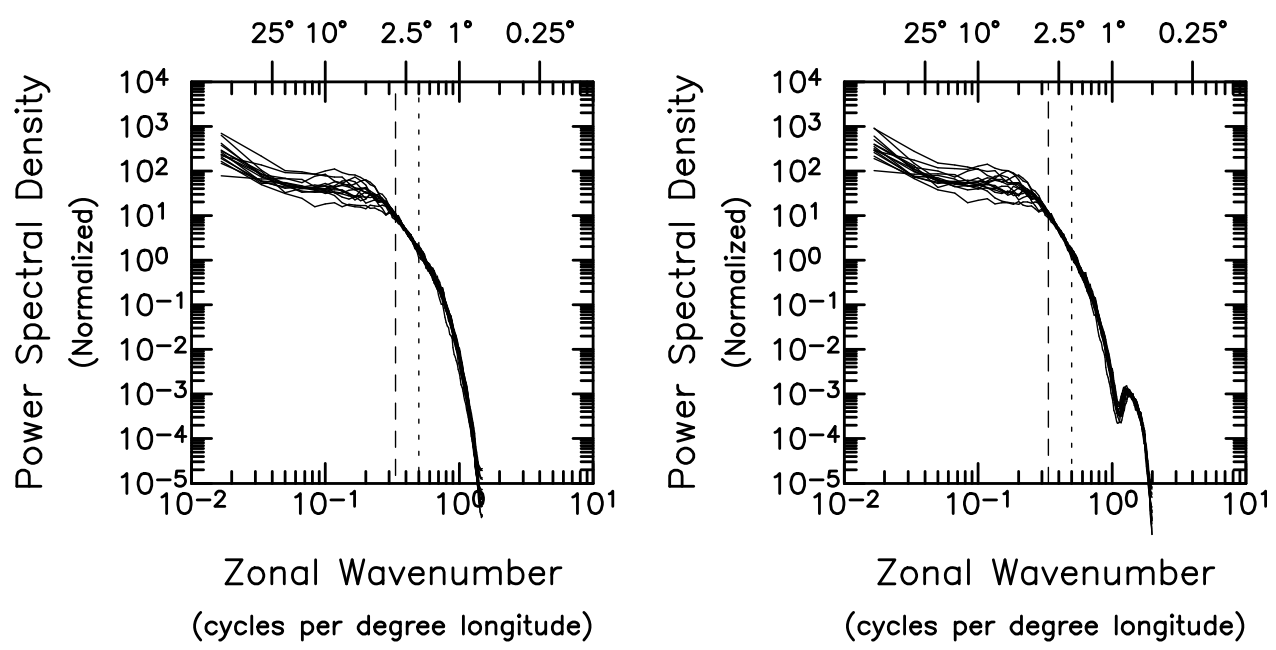

Figure 1. The zonal wave number spectra of sea surface height (left) from the AVISO $1 / 3^{\circ}$ Mercator gridded product and (right) from the AVISO $1 / 4^{\circ}$ product. See text for discussion of normalization. The vertical dashed and dotted lines correspond to wavelengths of $3^{\circ}$ and $2^{\circ}$, respectively.

implies in turn that $F_{8}\left(c_{n}\right)$ also equals zero. We are then left with

$$
\begin{aligned}
\frac{\partial \eta}{\partial r} \approx & F_{1}\left(c_{n}\right) \eta^{(1)}(r)+F_{3}\left(c_{n}\right) h^{2} \eta^{(3)}(r)+F_{5}\left(c_{n}\right) h^{4} \eta^{(5)}(r) \\
& +F_{7}\left(c_{n}\right) h^{6} \eta^{(7)}(r)+F_{9}\left(c_{n}\right) h^{8} \eta^{(9)}(r)
\end{aligned}
$$

where now each $F_{n}$ depends only on the four constants $c_{1}, c_{2}$, $c_{3}$, and $c_{4}$, since we have written $c_{-n}$ in terms of $c_{n}$ for $n=1$, $2,3,4$. Setting $F_{1}\left(c_{n}\right)=1$ and $F_{3}\left(c_{n}\right)=F_{5}\left(c_{n}\right)=F_{7}\left(c_{n}\right)=0$ provides four equations which can be solved for the four constants $c_{n}, n=1,2,3,4$ as shown in Table 1 . We are then left with $F_{9}\left(c_{n}\right) h^{8} \eta^{(9)}(r)$ as the leading-order error term. The formulae for second derivatives are obtained in like manner, the main difference being that the value of $\eta$ at the central grid point $r$ is utilized:

$$
\frac{\partial^{2} \eta}{\partial r^{2}} \approx \frac{1}{h^{2}} \sum_{n} c_{n} \eta(r+n h), \quad n=-4,-3,-2,-1,0,1,2,3,4
$$

\section{The von Neumann Analysis and Spectral Content of AVISO Data}

[11] We can anticipate some of the results of using wider stencils by following the "von Neumann analysis" given in section 9.6 of LeVeque [2007]. This analysis allows us to examine how accurate the stencil approximations for derivatives are, as a function of the differing length scales (wave numbers) contained in the spatially varying sea surface height, $\eta(r)$. We focus on the first derivative in this section.

[12] Because of the importance of the wave number content of the AVISO signal to the von Neumann analysis, we display in Figure 1 the wave number spectrum of AVISO. The figure displays the zonal wave number spectrum of sea surface height for wave number in units of cycles per degree of longitude, computed along 15 zonal sections in the North and South Pacific [see Chelton et al., 2011, Figure A2]. Spectra from both the $1 / 3^{\circ}$ Mercator grid AVISO product, and the $1 / 4^{\circ}$ AVISO product, are shown. The spectra have been normalized so that each spectrum has the same variance integrated over wavelengths shorter than $3^{\circ}$ (wave numbers higher than 0.333 cycles per degree of longitude). As discussed by Chelton et al. [2011], while spectra for wavelengths larger than $2^{\circ}-3^{\circ}$ display substantial variance from one section to the next (note the scatter of the different curves in the left-hand side of both Figures 1 (left) and 1 (right)), spectra for the different sections over wavelengths shorter than $2^{\circ}-3^{\circ}$ lie almost on top of each other. Chelton et al. [2011] argue that this demonstrates that the filtering inherent in the creation of AVISO products removes most of the variance in wavelengths shorter than $2^{\circ}-3^{\circ}$. We will refer to the $2^{\circ}-3^{\circ}$ scale as the wavelength resolution limit. The bi-linear interpolation introduces a sidelobe with peak at 1.333 cycles per degree into the zonal wave number spectrum of kinetic energy on the $1 / 4^{\circ}$ grid (compare Figure 1 (right) to Figure 1 (left)). The sidelobe is absent in spectra computed on the $1 / 3^{\circ}$ grid [Chelton et al., 2011] and is therefore an artifact introduced by interpolation.

[13] Since the sea surface height field $\eta$ on a grid $r$ can be written as a Fourier sum

$$
\eta(r)=\sum_{k} \hat{\eta}(k) e^{i k r}
$$

where $i=\sqrt{-1}$, it suffices to examine the derivative of the function $e^{i k r}$. This function is an eigenfunction of the "ideal differentiator" $\frac{\partial}{\partial r}$, with eigenvalue $\Lambda=i k$, since $\frac{\partial}{\partial r} e^{i k r}=$ $i k e^{i k r}$. Let us now examine the stencil approximation for the derivative of $\eta=e^{i k(r+n h)}$, the discretized equivalent of $e^{i k r}$. As before, $n$ is the index of the grid point number relative to the central grid point $r$, and $h$ is the grid point spacing. The wave number $k=\frac{2 \pi}{\lambda}$, where $\lambda$ is wavelength. Let $\Delta$ be the $2 m+1$ point stencil centered difference operator. Then we have

$$
\Delta \eta(r)=\frac{1}{h} \sum_{n=-m}^{m} c_{n} \eta(r+n h) \quad(n \neq 0)
$$



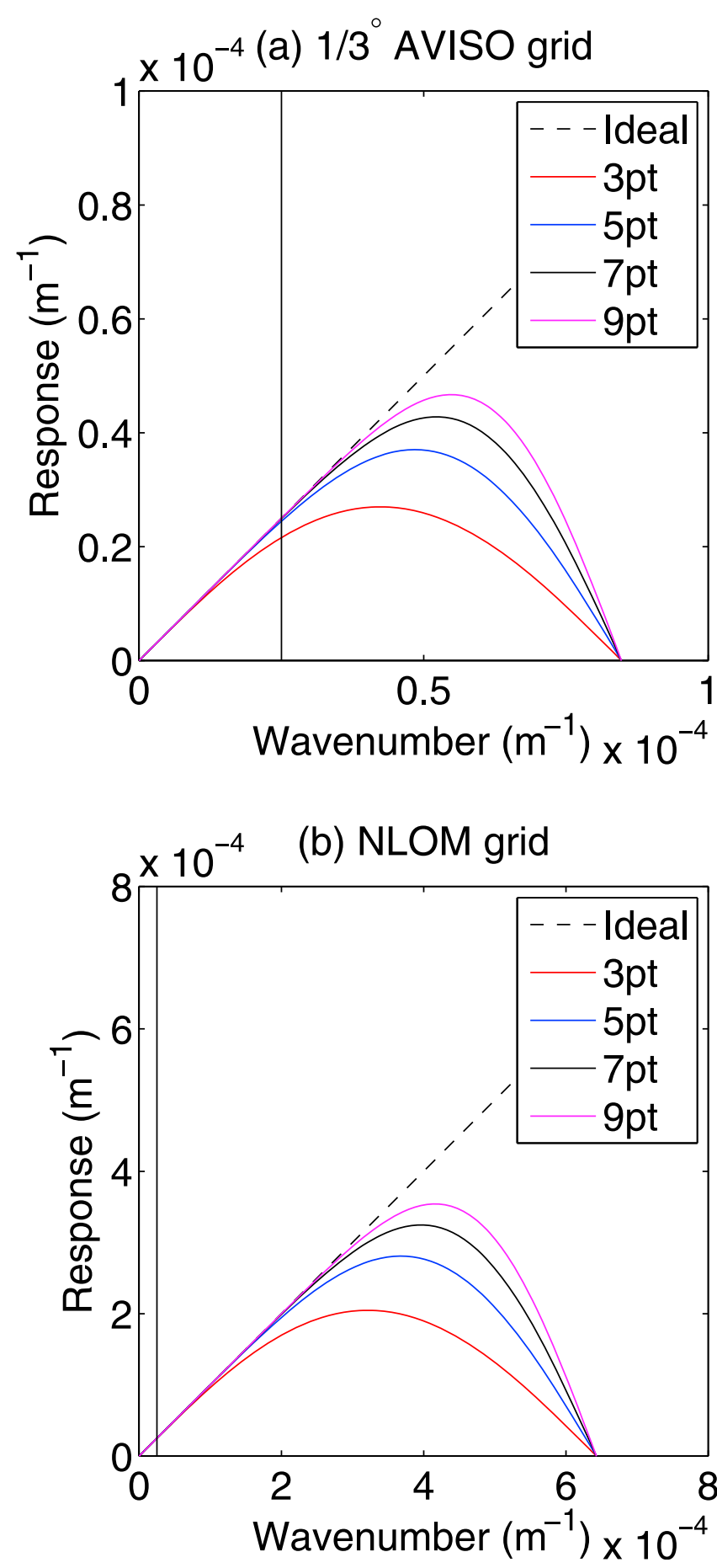

Figure 2. Response of an ideal differentiator and of 3-, 5-, 7-, and 9-point stencils, as a function of wave number, on (a) $1 / 3^{\circ}$ AVISO grid and (b) NLOM grid. The grid spacings used for Figures $2 \mathrm{a}$ and $2 \mathrm{~b}$ are 37 and $4.9 \mathrm{~km}$, respectively, and the response is the absolute value $|\Lambda|$ of the eigenvalue of the von Neumann analysis. See text for more details. Vertical lines are drawn at the wave number $(40 \mathrm{~km})^{-1}$, corresponding approximately to the $2-3^{\circ}$ wavelength resolution limitation of the AVISO sea surface height fields. or, since $c_{-n}=-c_{n}$,

$$
\Delta \eta(r)=\frac{1}{h} \sum_{n=1}^{m} c_{n}\left[e^{i k(r+n h)}-e^{i k(r-n h)}\right] .
$$

Therefore

$$
\Delta \eta(r)=\frac{1}{h} e^{i r k} \sum_{n=1}^{m} c_{n}[2 i \sin (n h k)] .
$$

Therefore $e^{i r k}$ is an "eigengridfunction" of the operator $\Delta$, with eigenvalue

$$
\Lambda=\frac{2 i}{h} \sum_{n=1}^{m} c_{n} \sin (n h k)
$$

For example, for a 3-point stencil, $m=1, c_{1}=\frac{1}{2}$, and $\Lambda=$ $\frac{i}{h} \sin (h k)$. For small $h k$ we can write $\Lambda \approx \frac{i}{h}\left[h k-\frac{1}{6}(h k)^{3}+\ldots\right] \approx$ $i k\left[1-\frac{1}{6}(h k)^{2}+\ldots\right]$. This agrees with the eigenvalue $i k$ of $\frac{\partial}{\partial r}$ to order $(h k)^{2}$.

[14] In Figure 2 we plot the results of the von Neumann analysis for classic centered differences. We plot the "response" $|\Lambda|$ for the 3-point, 5-point, 7-point, and 9-point stencils, as a function of wave number $k$. We also plot $k$, the value of $|\Lambda|$ for the ideal differentiator $\frac{\partial}{\partial r}$. The "response", represented by the vertical axis in figures like this, is sometimes referred to as the "filter transfer function" in the signal processing literature. In Figure $2 \mathrm{a}$ we set $h=37 \mathrm{~km}$, the equatorial grid spacing in the $\mathrm{x}$ - and $\mathrm{y}$-directions on the $1 / 3^{\circ}$ AVISO grid. In Figure $2 \mathrm{~b}$ we set $h=4.9 \mathrm{~km}$, the equatorial grid spacing in the $\mathrm{X}$-direction on the NLOM grid. As a result, note that the $x$-axes in Figures $2 a$ and $2 b$ differ from each other. In both plots we draw in $(40 \mathrm{~km})^{-1}$, the approximate wave number corresponding to the $\sim 2^{\circ}-3^{\circ}$ wavelength resolution limitation of the AVISO sea surface height fields (Figure 1) [Chelton et al., 2011], as a vertical line. Figure 2a shows that for wave numbers just below (length scales just above) the resolution limitation, the 3-point stencil deviates significantly from the ideal response. This implies that an important part of the signal is lost when 3-point stencils are used. The wider stencils do not deviate from the ideal differentiator until larger wave numbers (smaller scales) are reached. From this we anticipate that there will be substantial differences between derivatives computed using 3-point versus wider stencils on the $1 / 3^{\circ}$ AVISO grid (and on the $1 / 4^{\circ}$ AVISO grid, since the grid spacings are comparable). In Figure $2 b$, because of the smaller grid spacing, the wave number $(40 \mathrm{~km})^{-1}$ lies far below the wave numbers where the 3-point stencil and other stencils deviate from the ideal differentiator. For features with length scales larger than about $40 \mathrm{~km}$, we therefore expect stencil width to make less of a difference on the high-resolution NLOM grid than on the coarser AVISO grid.

[15] To illustrate the implications of the von Neumann analysis, we utilized results from the idealized two-layer doubly periodic pseudo-spectral quasi-geostrophic turbulence simulations of Arbic and Flierl [2004]. Here we used the snapshot in their Figure $8 \mathrm{~d}$. The advantage of using the 


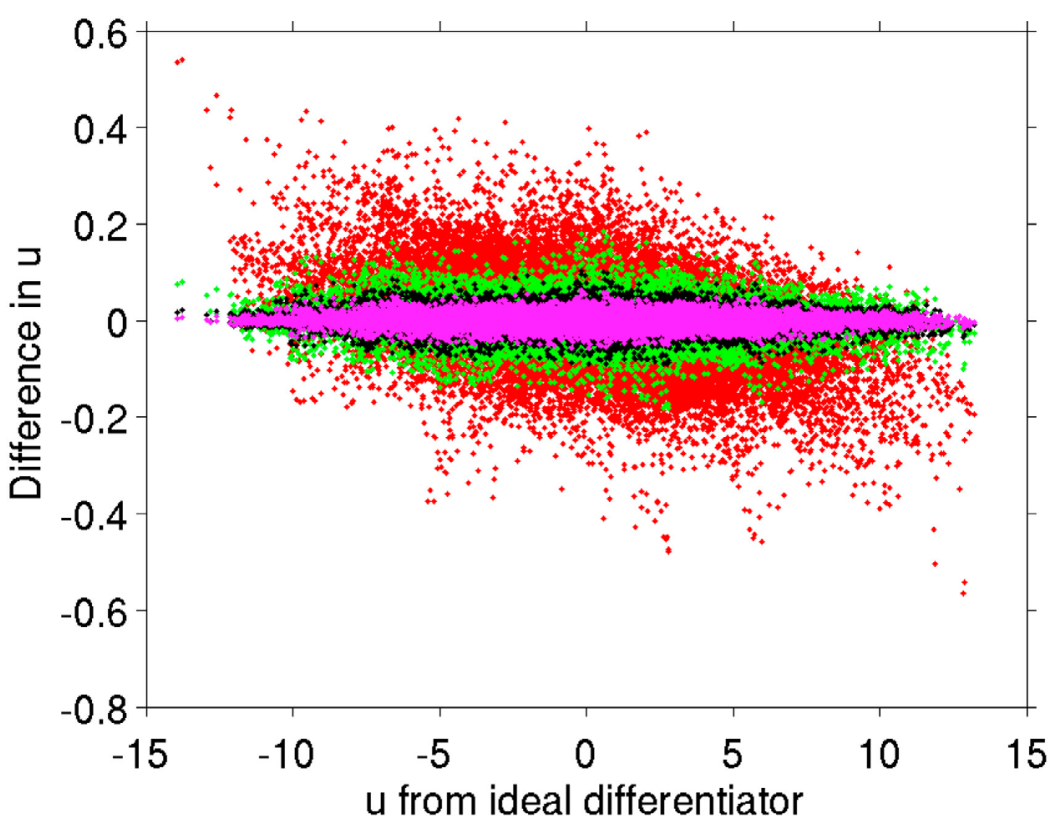

Figure 3. Differences between zonal velocity component $u$ computed using 3-point, 5-point, 7-point, and 9-point stencils, and $u$ computed using an "ideal differentiator" spectral technique (see text). Velocities are computed from a snapshot of the upper layer stream function in an idealized doubly periodic quasigeostrophic turbulence model. The y-axis of the scatterplot is the difference between $u$ computed on stencils and $u$ computed from the ideal differentiator, while the x-axis represents $u$ computed from the ideal differentiator. Red, green, black, and magenta dots represent differences computed using 3-point, 5-point, 7-point, and 9-point stencils, respectively. Both axes are normalized by the imposed time-mean flow in the idealized model, which was taken to be $1 \mathrm{~cm} \mathrm{~s}^{-1}$ in the work of Arbic and Flierl [2004].

idealized model in this illustration is that velocities are computed in this model via the "ideal differentiator" of the von Neumann analysis. (Indeed, this property is one reason pseudo-spectral models have enjoyed a long usage.) In pseudo-spectral models, the model stream function is Fourier transformed, multiplied by $-i l$ or $i k$, and then inverse transformed to obtain $u$ and $v$, respectively, where $l$ is the meridional wave number and $k$ is the zonal wave number. Since the idealized model is doubly periodic, no tapering near the edges is required to perform this computation, in contrast to spectral computations done in more realistic domains.

[16] Figure 3 displays scatterplots of the differences $u_{\text {stencil }}-u_{\text {ideal }}$ between zonal velocity $u_{\text {stencil }}$ computed from the idealized model using 3-point, 5-point, 7-point, and 9-point stencils, and $u_{\text {ideal }}$ computed using the "ideal differentiator" spectral technique actually employed in the idealized model. The differences $u_{\text {stencil }}-u_{\text {ideal }}$ are plotted on the $\mathrm{y}$-axis of Figure 3 , while the x-axis displays $u_{\text {ideal }}$ computed from the ideal differentiator. The differences decrease as stencil width increases, consistent with the von Neumann analysis discussed above. Because the dynamics in this particular simulation of the idealized model are nearly isotropic, and because the grid is also isotropic, results for the meridional velocity $v$ are nearly identical to those in Figure 3, and are not shown for the sake of brevity. Because velocities computed from the 9-point stencil lie closer to velocities computed from the ideal differentiator than do velocities computed from narrower stencils, we will take the 9-point stencil results as our standard in what follows. Ideally, we will expect 7-point stencil results to lie closer to 9-point stencil results than 5-point stencil results do, and 5-point stencil results to lie closer to 9-point stencil results than 3-point stencil results do.

\section{Effect of Stencil Width on Geostrophic Velocity Estimates From Altimeter Data and Realistic Models}

[17] In this section we examine the impact of stencil width on geostrophic velocity estimates computed using classic centered differences from a single snapshot of sea surface height. We utilize the AVISO weekly reference (two satellite) product for November 5, 2008. Both zonal and meridional velocity components are computed, and results from differentiating on both the $1 / 3^{\circ}$ and $1 / 4^{\circ}$ AVISO grids are shown. The $1 / 3^{\circ}$ AVISO grid has 915 by 1080 grid points in the north-south and east-west directions, respectively, while the $1 / 4^{\circ}$ AVISO grid has 721 by 1440 grid points. We also utilize the February 15, 2002 snapshot of sea surface height from NLOM, which has 4384 by 8192 grid points on a nearglobal domain $\left(72^{\circ} \mathrm{S}\right.$ to $\left.65^{\circ} \mathrm{N}\right)$. The NLOM grid point spacing is $1 / 32^{\circ}$ in the north-south direction and $45 / 1024^{\circ}$ in the east-west direction. In this and succeeding results sections, we choose to emphasize the deep ocean, in both the AVISO and NLOM calculations. To do so, we display only velocities computed at grid points for which the seafloor depth exceeds $1000 \mathrm{~m}$. Furthermore, where comparisons are made between $u$ and $v$ or between velocity components computed from 
(a) $u, 1 / 3$ degree

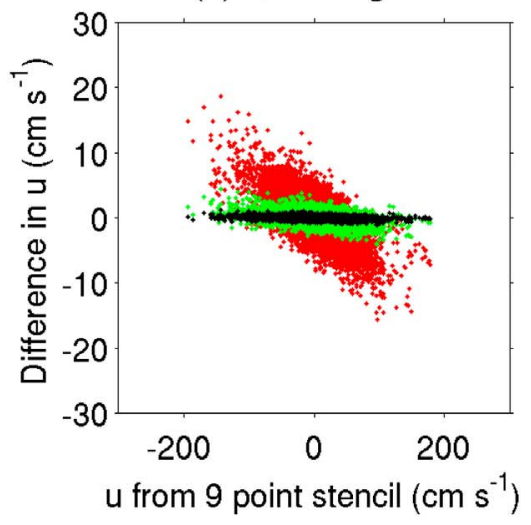

(b) $v, 1 / 3$ degree

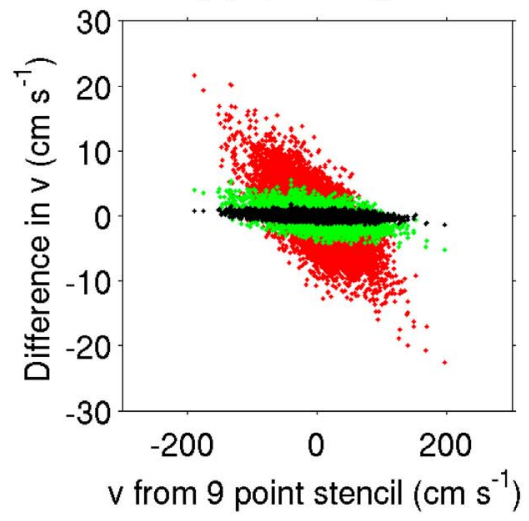

(c) $u, 1 / 4$ degree

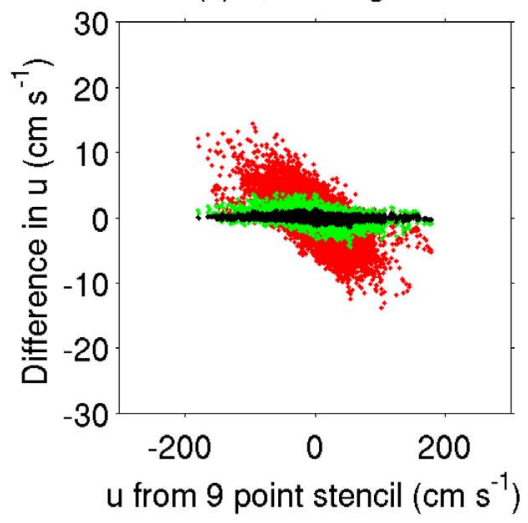

(d) $v, 1 / 4$ degree

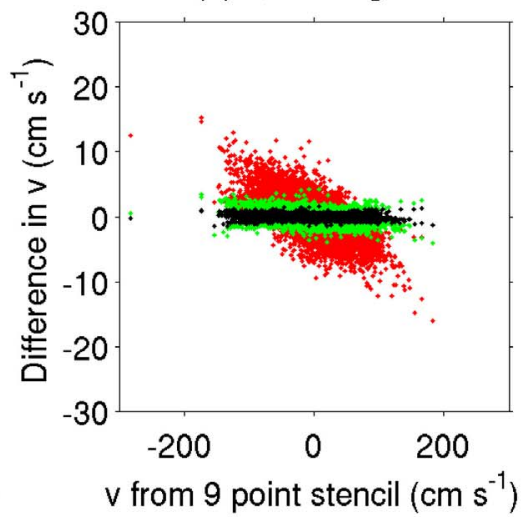

Figure 4. Scatterplots of velocity components computed from 3-point, 5-point, and 7-point stencils minus components computed from 9-point stencils (y-axis), plotted versus components computed from 9-point stencils (x-axis). Red, green, and black dots denote results with 3-point, 5-point, and 7-point stencils, respectively. (a) Zonal velocity $u$ computed on the $1 / 3^{\circ}$ Mercator grid reference AVISO product for November 5, 2008. (b) As in Figure 4a but for meridional velocity $v$. (c) As in Figure 4a but for zonal velocity $u$ computed on the $1 / 4^{\circ}$ latitude-longitude grid reference AVISO product for November 5, 2008. (d) As in Figure 4c but for meridional velocity $v$.

different stencil widths, we only display points for which all relevant velocity components are defined. For wider stencils, we will lose points near boundaries, where a full span of the stencil does not exist because of land.

[18] Figure 4 shows scatterplots of geostrophic velocity components computed from the aforementioned AVISO products. In like manner to Figure 3 , we plot $u_{\text {narrow }}-u_{9 p t}$ versus $u_{9 p t}$ and $v_{\text {narrow }}-v_{9 p t}$ versus $v_{9 p t}$, where the " $9 p t$ " subscript denotes a 9-point stencil difference and "narrow" denotes either a 3-point, 5-point, or 7-point stencil difference. As anticipated in the previous section, whereas the differences between 7-point and 9-point stencil results (black dots) lie close to zero for all grid points, the differences between 3-point and 9-point stencil results (red dots) lie farther from zero (display much more scatter). The scatter is not distributed around zero evenly, but rather reveals what we will call a "speed-dependent bias". By this we mean that where the 9-point stencil results are negative, the 3-point minus 9-point stencil results are positive, and vice versa. The difference between 5-point and 9-point stencil results (green dots) displays less speed-dependent bias than the 3-point minus 9-point results but more speed-dependent bias than the 7-point minus 9-point results. The speed-dependent bias at individual grid points can be as large as $10 \%$ or more. These behaviors are seen for both the $u$ and $v$ components of velocity, and for both velocities computed on the $1 / 3^{\circ}$ AVISO grid and the $1 / 4^{\circ}$ AVISO grid. A stochastic model for the speed-dependent bias will be presented in section 7 .

[19] Figure 5 demonstrates that similar behaviors are seen in geostrophic velocities computed through differentiation of the sea surface heights in NLOM. Here we compute geostrophic velocities on both the native 4384 by 8192 NLOM 
(a) $\mathrm{u}$, native NLOM grid

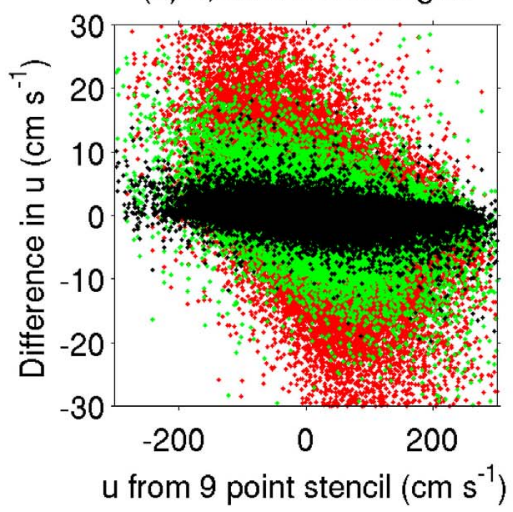

(b) $v$, native NLOM grid

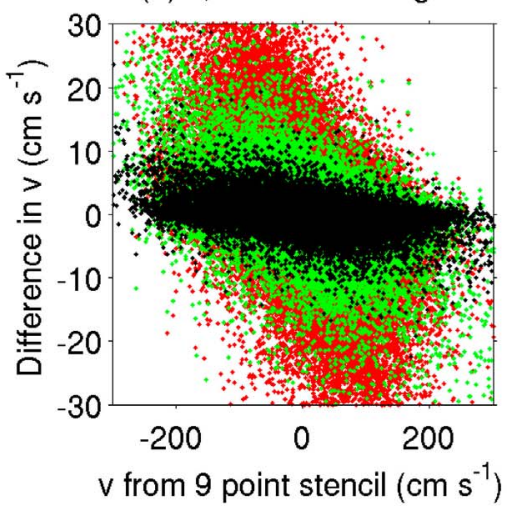

(c) u, NLOM grid decimated by 8

(d) V, NLOM grid decimated by 8
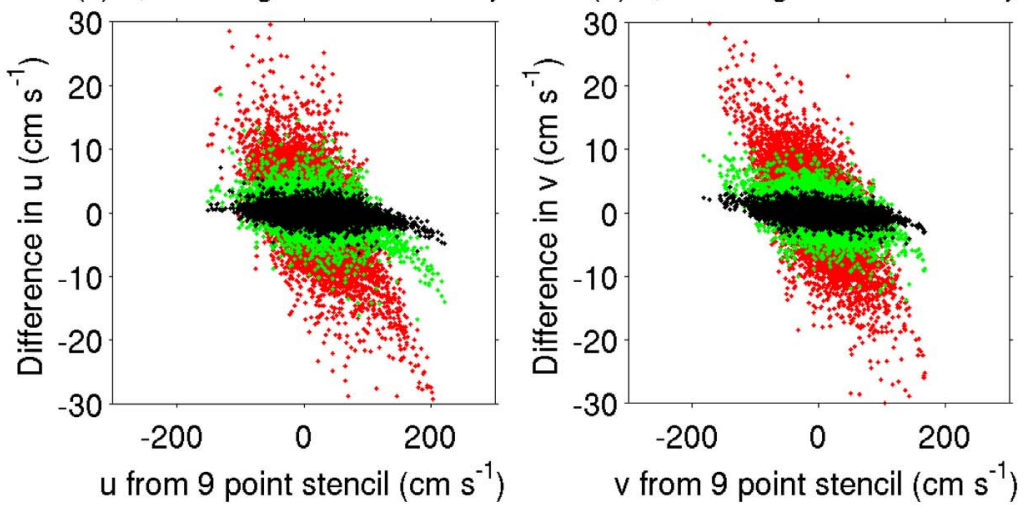

Figure 5. As in Figure 4, but using February 15, 2002 output from the Naval Research Laboratory Layered Ocean Model (NLOM) rather than gridded altimeter data. (a) Zonal velocity $u$ and (b) meridional velocity $v$ computed on NLOM output on the native 4384 by 8192 grid. (c) Zonal velocity $u$ and (d) meridional velocity $v$ computed on NLOM output decimated by a factor of 8 in both directions to a 548 by 1024 grid.

grid and on a grid that is decimated by a factor of 8 in both directions, to a 548 by 1024 grid that is comparable in resolution $\left(1 / 4^{\circ}\right.$ in the north-south direction, $0.35^{\circ}$ in the east-west direction) to the AVISO grids. In the case of the decimated grid only the subsampled grid points are used in the calculation, so that the grid spacing is 8 times larger than on the original grid. The 3-point stencil differences are significantly different from 7-point and 9-point differences, and display a speed-dependent bias, on the high resolution grid as well as on the decimated grid. At some grid points the difference between 3-point and 9-point estimates are as large as $10 \%$. Results from the stochastic model in section 7 are consistent with this suggestion of a speed-dependent bias in 3 -point stencil results even in the limit of high resolution. The smaller scatter (i.e. the smaller discrepancies seen between narrow stencil results and 9-point stencil results) seen in Figure 4 relative to Figure 5 is consistent with the weak signal in the AVISO data at scales smaller than the $2-3^{\circ}$ wavelength resolution limitation (Figure 1) [Chelton et al., 2011].
[20] In Figure 6 we display the zonally averaged discrepancies between the squares of zonal velocity components computed from AVISO using stencils of differing width:

$$
D_{u}=\frac{\left[u_{\text {narrow }}^{2}-u_{9 p t}^{2}\right]}{\left[u_{9 p t}^{2}\right]},
$$

where [] represents a zonal average operator. We also display

$$
D_{v}=\frac{\left[v_{\text {narrow }}^{2}-v_{9 p t}^{2}\right]}{\left[v_{9 p t}^{2}\right]}
$$

the analogous quantity for $v$ (meridional velocity). In all cases we find that the $D$ values lie much closer to zero for 7-point stencil results than for 3-point stencil results, with 5-point stencil results lying in between these extremes. This demonstrates a "convergence" in the calculation of velocities as stencil width increases. For both $u$ and $v$ and on both the 
(a) $u, 1 / 3$ degree
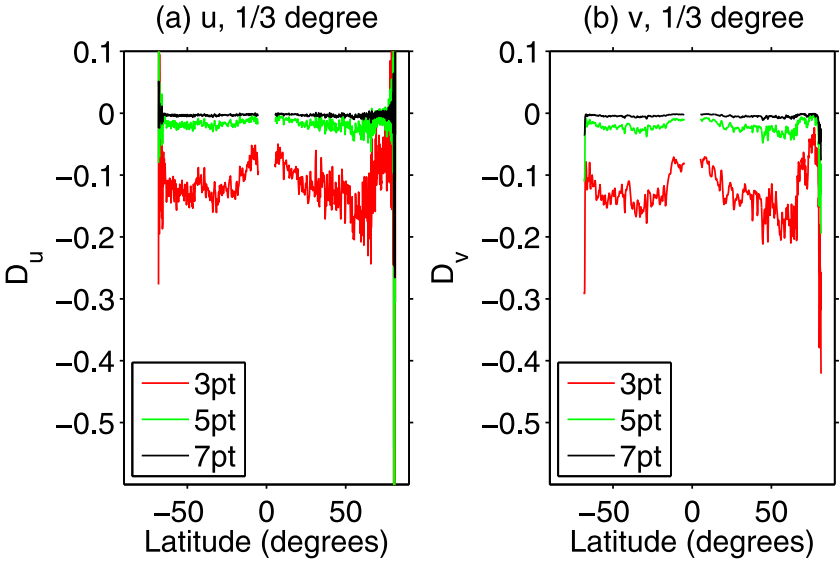

(c) u, 1/4 degree

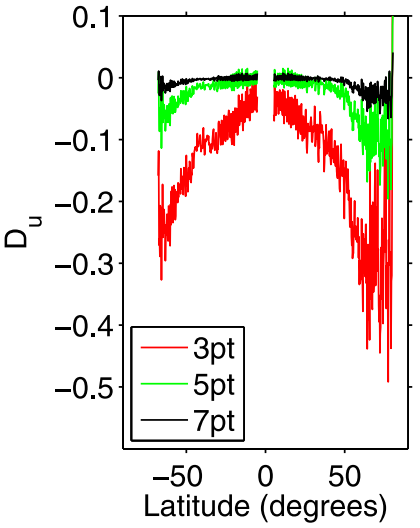

(d) $v, 1 / 4$ degree

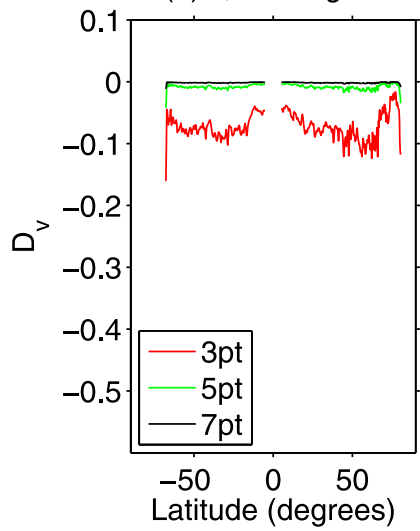

Figure 6. Values of $D_{u}$ and $D_{v}$, the zonally averaged discrepancies between the squared velocity components computed from 3-, 5-, and 7-point stencils and those computed from 9-point stencils. (a) $D_{u}$ values computed on the $1 / 3^{\circ}$ Mercator grid reference AVISO product for November 5, 2008. (b) As in Figure 6a but for $D_{v}$. (c) As in Figure 6 a but for $1 / 4^{\circ}$ latitudelongitude grid reference AVISO product for November 5 , 2008. (d) As in Figure 6c but for $D_{v}$.

$1 / 3^{\circ}$ and $1 / 4^{\circ}$ grids, the 3 - and 5 -point stencils yield velocity variances that are too weak with respect to the 9-point stencils, over most latitudes, consistent with the speed-dependent biases seen in Figure 4. On the isotropic $1 / 3^{\circ}$ Mercator grid, the values of $D_{u}$ and $D_{v}$ are comparable, with typical values of -0.1 for the 3 -point stencil. On the anisotropic $1 / 4^{\circ}$ latitude-longitude grid, the 3-point stencil $D_{u}$ values are as low as -0.2 to -0.3 in mid- and high-latitudes, whereas $D_{v}$ values seldom drop below -0.1 . This is consistent with the fact that the y-spacing on the anisotropic grid remains relatively wide at high latitudes, unlike the x-spacing at high latitudes on the anisotropic grid, or the spacing in either direction at high latitudes on the isotropic grid (recall that the error estimates increase with increasing grid spacing). On the anisotropic $1 / 4^{\circ}$ grid, increasing the stencil width reduces not only the $D$ values, but also the difference between values of $D_{u}$ versus $D_{v}$.

[21] It should be noted that some of the differences between results on the $1 / 4^{\circ}$ versus $1 / 3^{\circ}$ AVISO grid may be due to the bi-linear interpolation from the latter to the former, as well as to the differences in the two grid spacings. As

noted earlier, the bi-linear interpolation introduces an artificial sidelobe into the zonal wave number spectrum of kinetic energy on the $1 / 4^{\circ}$ grid. To investigate the effects of interpolation further, we have interpolated the idealized model snapshot discussed in section 3 to coarser grids, and we find that the difference between narrow and wide stencil estimates increases over that found on the original higher resolution grid. Interpolation of the idealized model output to a coarser grid which anisotropic (more widely spaced in one direction than the other) yields a greater sensitivity to stencil width for derivatives computed in the widely spaced direction than for derivatives computed in the other (better-resolved) direction. All of this is consistent with the results in Figure 6.

[22] Figures 7 and 8 display $D_{u}$ and $D_{v}$ values computed from NLOM. We show results computed on the original 4384 by 8192 grid as well as on this grid decimated by factors of 2,4 , and 8 in both horizontal directions. As in the AVISO results, the difference between narrow stencil results and 9-point stencil results decreases rapidly as the stencil width increases, for both velocity components. The disparity between 3-point stencil results and wider stencil results increases as the grid spacing increases. The results for both (a) $u$, native NLOM grid

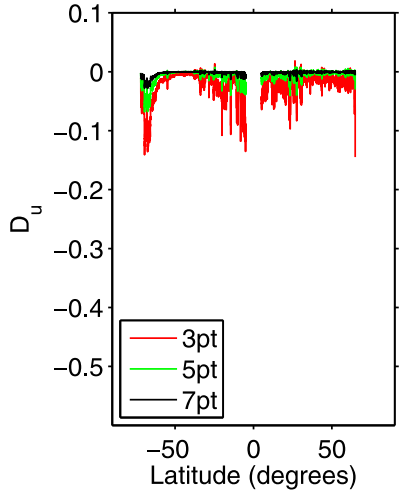

(b) $v$, native NLOM grid

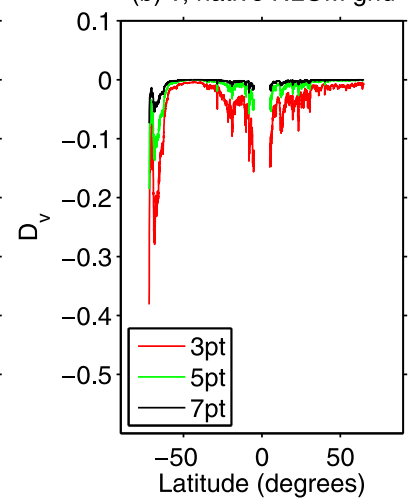

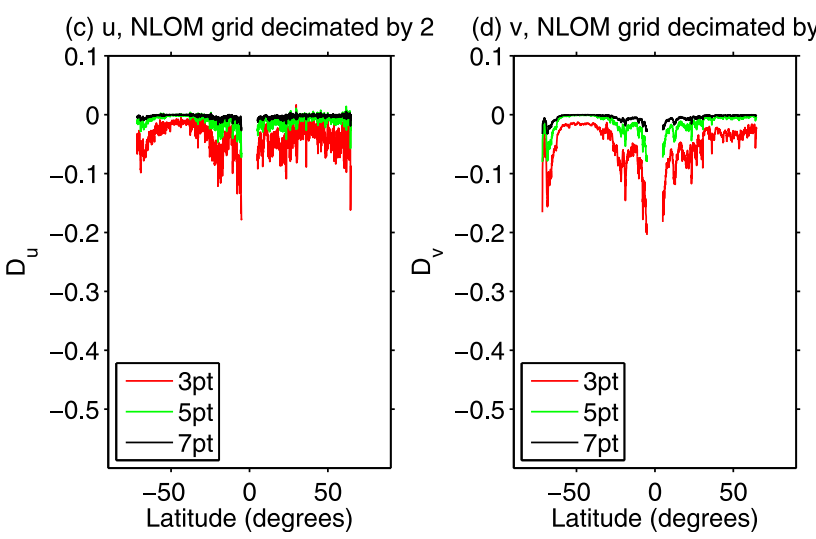

Figure 7. As in Figure 6, but using February 15, 2002 NLOM output rather than gridded altimeter data. Values of $D_{u}$ and $D_{v}$, the zonally averaged discrepancies between the squared velocity components computed from 3-, 5-, and 7-point stencils and those computed from 9-point stencils. (a) $D_{u}$ and (b) $D_{v}$ computed from NLOM output on a 4384 by 8192 grid. (c) $D_{u}$ and (d) $D_{v}$ computed on NLOM output decimated by a factor of 2 in both directions. 

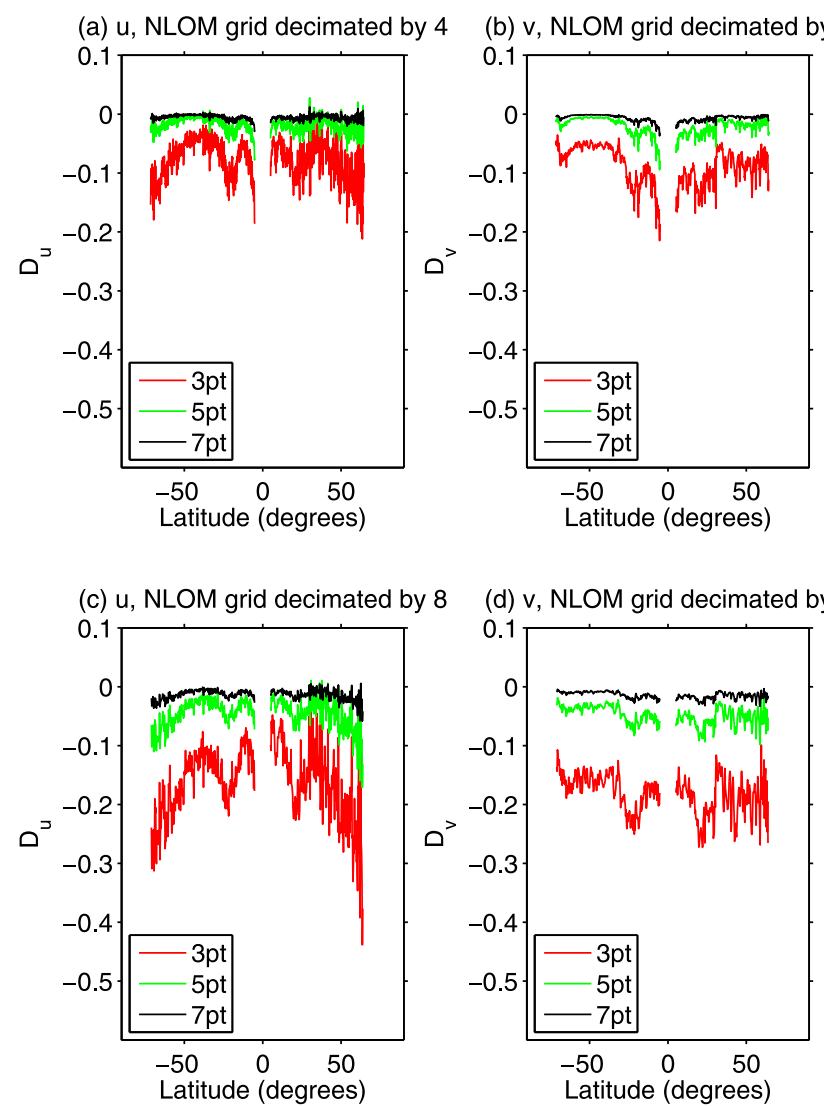

Figure 8. As in Figure 7, but for (a and b) NLOM grids decimated by a factor of 4 in both directions, and for (c and d) decimation by a factor of 8 in both directions.

$D_{u}$ and $D_{v}$ on the coarsest grid shown (decimation by factor of 8; Figures 8c and 8d) are remarkably similar to the results on the AVISO grids, especially the AVISO $1 / 4^{\circ}$ grid. In all of the latter cases, the 3-point stencil $D_{u}$ and $D_{v}$ values can reach as low as -0.1 to -0.3 . Results very similar to those in Figures 8c and 8d are obtained by smoothing the highresolution NLOM results onto a grid with eight times lower resolution, rather than by subsampling (decimation). For the sake of brevity the smoothed results are not shown here. Having used the high-resolution model to demonstrate that sensitivity to stencil width is not merely an artifact of the data processing inherent in the creation of gridded altimeter products, we focus only on analysis of altimeter data in the remainder of the paper.

\section{Effects of Non-uniform Grid Spacing}

[23] The formulae in section 2 have assumed that the grid spacing as measured in kilometers is uniform along a particular direction of interest, though it may vary between the $\mathrm{x}$ - and $\mathrm{y}$-directions. However, on the $1 / 3^{\circ}$ AVISO grid, the grid spacing as measured in kilometers in the $y$-direction is non-uniform. Here we will describe a method which accounts for non-uniform grid spacing such as this in computations using classic centered differences.

[24] Suppose we are interested in a $2 m+1$ point stencil on a non-uniform grid. As in section 2, let the central point, where the derivative is to be estimated, be $r$. Let the coordinates of the grid points in the stencil be $r+h_{m}$, $r+h_{m-1}, \ldots, r+h_{1}, r, r-h_{-1}, \ldots, r-h_{-(m-1)}, r-h_{-m}$. Let us expand in Taylor series

$$
\eta\left(r+h_{n}\right)=\eta(r)+h_{n} \eta^{(1)}(r)+\frac{h_{n}^{2} \eta^{(2)}(r)}{2 !}+\ldots+\frac{h_{n}^{p} \eta^{(p)}(r)}{p !}+\ldots
$$

and

$$
\begin{aligned}
\eta\left(r-h_{-n}\right)= & \eta(r)-h_{-n} \eta^{(1)}(r)+\frac{h_{-n}^{2} \eta^{(2)}(r)}{2 !}+\ldots \\
& +\frac{(-1)^{p} h_{-n}^{p} \eta^{(p)}(r)}{p !}+\ldots
\end{aligned}
$$

We want to find coefficients $c_{n}$ such that

$$
\begin{aligned}
& c_{m} \eta\left(r+h_{m}\right)+c_{m-1} \eta\left(r+h_{m-1}\right)+\ldots+c_{1} \eta\left(r+h_{1}\right) \\
& \quad+c_{0} \eta(r)+c_{-1} \eta\left(r-h_{-1}\right)+\ldots+c_{-(m-1)} \eta\left(r-h_{-(m-1)}\right) \\
& \quad+c_{-m} \eta\left(r-h_{-m}\right) \approx \eta^{(1)}(r),
\end{aligned}
$$

to as high an order as the stencil width allows. For example, for a 3-point stencil, solving the matrix equation

$$
\left(\begin{array}{ccc}
1 & 1 & 1 \\
h_{1} & 0 & -h_{-1} \\
h_{1}^{2} & 0 & h_{-1}^{2}
\end{array}\right)\left(\begin{array}{c}
c_{1} \\
c_{0} \\
c_{-1}
\end{array}\right)=\left(\begin{array}{l}
0 \\
1 \\
0
\end{array}\right)
$$

or

$$
\left(\begin{array}{c}
c_{1} \\
c_{0} \\
c_{-1}
\end{array}\right)=\left(\begin{array}{ccc}
1 & 1 & 1 \\
h_{1} & 0 & -h_{-1} \\
h_{1}^{2} & 0 & h_{-1}^{2}
\end{array}\right)^{-1}\left(\begin{array}{l}
0 \\
1 \\
0
\end{array}\right)
$$

yields the solution

$$
\begin{gathered}
c_{1}=\frac{h_{-1}}{h_{1}\left(h_{1}+h_{-1}\right)}, \\
c_{0}=\frac{h_{1}-h_{-1}}{h_{1} h_{-1}}, \\
c_{-1}=\frac{-h_{1}}{h_{-1}\left(h_{1}+h_{-1}\right)},
\end{gathered}
$$

with leading order error term $\frac{h_{1} h_{-1} \eta^{(3)}(r)}{6}$. These formulae collapse to the uniform grid formulae in the case $h_{1}=h_{-1}$. In the y-direction on the $1 / 3^{\circ}$ AVISO grid, where $h_{1}$ and $h_{-1}$ are slightly unequal, $c_{0}$ will be small but not zero, and $c_{1}$ and $c_{-1}$ will be nearly equal but not exactly so. For a 5-point stencil on a non-uniform grid the coefficients $c_{n}$ are given by

$$
\left(\begin{array}{c}
c_{2} \\
c_{1} \\
c_{0} \\
c_{-1} \\
c_{-2}
\end{array}\right)=\left(\begin{array}{ccccc}
1 & 1 & 1 & 1 & 1 \\
h_{2} & h_{1} & 0 & -h_{-1} & -h_{-2} \\
h_{2}^{2} & h_{1}^{2} & 0 & h_{-1}^{2} & h_{-2}^{2} \\
h_{2}^{3} & h_{1}^{3} & 0 & -h_{-1}^{3} & -h_{-2}^{3} \\
h_{2}^{4} & h_{1}^{4} & 0 & h_{-1}^{4} & h_{-2}^{4}
\end{array}\right)^{-1}\left(\begin{array}{l}
0 \\
1 \\
0 \\
0 \\
0
\end{array}\right)
$$

The 7- and 9-point stencil coefficients on a non-uniform grid are solved for in like manner.

[25] We have computed 3-, 5-, 7-, and 9-point stencil derivatives for $1 / 3^{\circ}$ AVISO data using these formulae for a non-uniform grid. The results are not shown for the sake of 
(a)

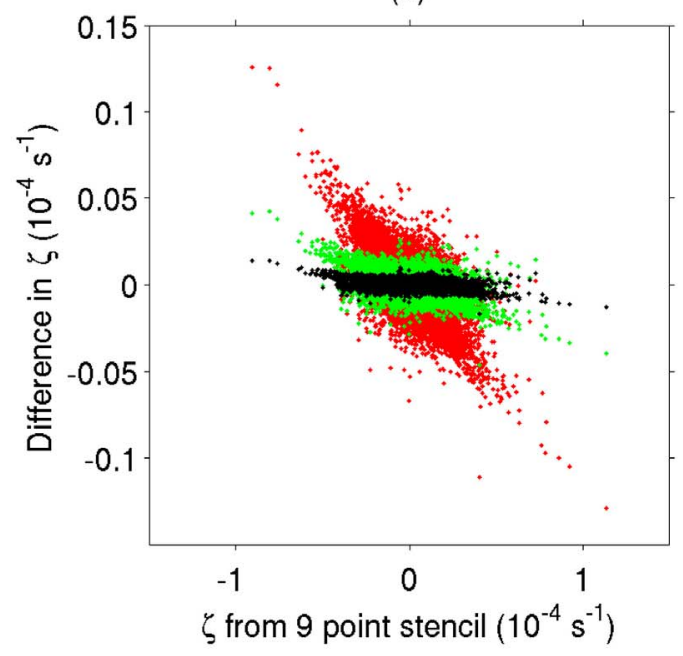

(b)

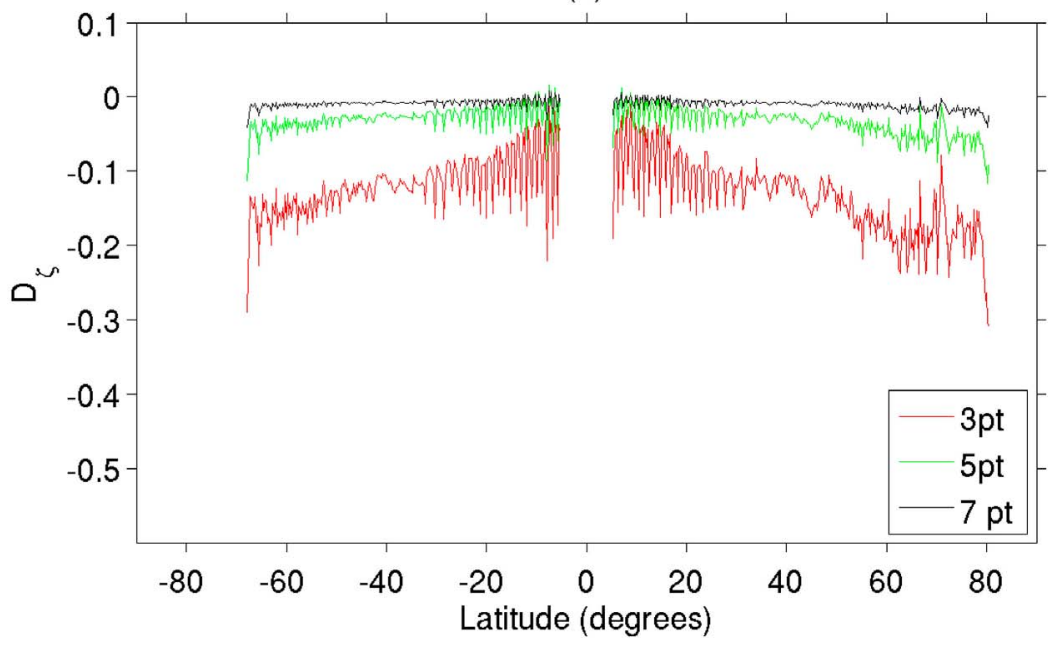

Figure 9. Effects of stencil width on estimates of geostrophic vorticity $\zeta$ computed on the $1 / 4^{\circ}$ latitudelongitude grid reference AVISO product for November 5, 2008. (a) Scatterplot of $\zeta$ computed from 3-point, 5-point, and 7-point stencils minus $\zeta$ computed from 9-point stencils (y-axis), plotted versus $\zeta$ computed from 9-point stencils (x-axis). Red, green, and black dots denote results obtained from 3-point, 5-point, and 7-point stencils, respectively. (b) Values of $D_{\zeta}$, the zonally averaged discrepancies between squared vorticities, computed from 3-, 5-, and 7-point stencils and those computed from 9-point stencils.

brevity. The differences between the zonal velocity component $u$ computed using the non-uniform grid procedure above versus that computed from uniform grid formulae are measurable but significantly smaller than the differences arising from different stencil widths. The equivalents of $D_{u}$ and $D_{v}$, for example, computed from differences between results using the non-uniform grid versus uniform grid formulae, are of order $1 \%$ for the AVISO $1 / 3^{\circ}$ Mercator grid, rather than of order $10-20 \%$ as seen with differing stencil widths. This result will differ for more rapidly changing grid spacing. For the sake of simplicity we will continue to use the uniform grid spacing formulae in the remainder of the paper.

\section{Effect of Stencil Width on Vorticity Estimates}

[26] In this section we briefly discuss the effects of stencil width on estimates of vorticity $\zeta$, computed using classic centered differences from the $1 / 4^{\circ}$ grid AVISO sea surface height reference product for November 5, 2008. In Figure 9a we show a scatterplot of $\zeta_{\text {narrow }}-\zeta_{9 p t}$ plotted versus $\zeta_{9 p t}$, where again "narrow" denotes either a 3-point, 5-point, or 7point stencil and "9pt" denotes a 9-point stencil. As in the scatterplots of velocity (Figures 4 and 5), the 7-point stencil result lies closer to the 9-point result than does the 5-point stencil result, and much closer than does the 3-point stencil result. In Figure 9b we display

$$
D_{\zeta}=\frac{\left[\zeta_{\text {narrow }}^{2}-\zeta_{9 p t}^{2}\right]}{\left[\zeta_{9 p t}^{2}\right]},
$$

where $\zeta_{\text {narrow }}$ is computed on the narrower 3-, 5-, and 7-point stencils, and $\zeta_{9 p t}$ is computed on the 9-point stencil. As in the plots of $D_{u}$ and $D_{v}$ (Figures 6-8), we see a decrease to 
near-zero $D$ values with increasing stencil width. Note that omitting the $\beta$ term (the last term on the right-hand side of (3)) in the estimate of $\zeta$ yields results that look virtually unchanged from those shown in Figure 9. Thus, as expected, the $\beta$ term does not play a first-order role in the computation of vorticity.

\section{A Stochastic Model for the Speed-Dependent Bias in Derivative Estimates}

[27] In Figures 4 and 5, we saw that the difference between "narrow" stencil and 9-point stencil estimates of geostrophic velocity is generally positive for negative values of $u$ or $v$, and negative for positive values. We referred to this in section 4 as a "speed-dependent bias". Figures 6-8 demonstrate that, as a result, values of $D_{u}$ and $D_{v}$ are generally negative, by an order of $10 \%$ for a stencil width of three points. Figure 9 demonstrates that the same principles hold for geostrophic vorticity estimates.

[28] Here we construct a stochastic model which qualitatively captures these biases in the narrow-stencil estimates made using classic centered differences. For simplicity, we discuss here only the first-derivative (velocity) results. We also consider the grid spacing $h$ to be constant since the results of section 5 show that the errors from the effects of the small latitudinal variation of grid spacing in the y-direction for isotropic grids are much smaller than the effects arising from the coarseness of the grid spacing. Our stochastic model is a simple first order auto-regressive model (AR1). We represent $\eta$ as

$$
\eta(r+h)=\phi \eta(r)+w(r+h)
$$

where $w(r+h)$ is a value taken from a purely random process $\mathrm{W}$, also called a "Gaussian white-noise-in-space stochastic process". That is, to each point in space $r$ we associate a stochastic process $\mathrm{W}$ giving values $w(r)$ that are completely uncorrelated with $w(r+\delta)$ at a neighboring point $r+\delta$ where $\delta \neq 0$. The parameter $0 \leq \phi<1$ determines the spatial autocorrelation of $\eta(r)$ and in the limit $\phi \rightarrow 0$ we see that $\eta$ approaches the Gaussian white-noise-in-space stochastic process. A similar equation to (24) applies at other points. In particular, assuming homogeneous statistics, we can write

$$
\begin{gathered}
\eta(r-h)=\phi \eta(r)+w(r-h) \\
\eta(r+2 h)=\phi \eta(r+h)+w(r+2 h) \\
\eta(r-2 h)=\phi \eta(r-h)+w(r-2 h) .
\end{gathered}
$$

Substituting these AR1 models into the formulae in Table 1, we find the following relation between the first derivatives computed with a 3 -point stencil (subscript $N=3$ below) and a 5-point stencil (subscript $N=5$ ):

$$
\begin{aligned}
\eta_{N=5}^{(1)}(r)= & \frac{8}{6} \eta_{N=3}^{(1)}(r)-\frac{\eta(r+2 h)-\eta(r-2 h)}{12 h} \\
= & \frac{8}{6} \eta_{N=3}^{(1)}(r)-\frac{\phi}{6} \frac{[\eta(r+h)-\eta(r-h)]}{2 h} \\
& -\frac{[w(r+2 h)-w(r-2 h)]}{12 h} \\
= & \frac{(8-\phi)}{6} \eta_{N=3}^{(1)}(r)+W^{\prime},
\end{aligned}
$$

where

$$
W^{\prime}=-\frac{[w(r+2 h)-w(r-2 h)]}{12 h}=\frac{\sqrt{2}}{12} \frac{W}{h} \approx 0.1179 \frac{W}{h} .
$$

Here we have used the fact that $w(r+2 h)$ and $w(r-2 h)$ are uncorrelated, so that their sum is another random function with a magnitude obtained from quadrature (i.e., taking the square root of the sum of the squares). Inspection of this relationship reveals that the 5-point stencil estimate is equal to a constant $\frac{8-\phi}{6}$ times the 3 -point stencil estimate, plus a purely random process $W^{\prime}$. It is important to note that $\mathrm{W}^{\prime}$ is uncorrelated with the 3-point stencil estimate since it is the linear combination of $w$ from points outside the 3-point stencil. Since $\frac{8-\phi}{6}$ exceeds one, the $N=3$ derivative displays a speed-dependent bias relative to the more accurate $N=5$ stencil; when the derivative is positive the discrepancy is negative and vice versa.

[29] Similar manipulations yield

$$
\begin{aligned}
\eta_{N=7}^{(1)}(r)= & \frac{45}{30} \eta_{N=3}^{(1)}(r)-\frac{9}{30} \frac{\eta(r+2 h)-\eta(r-2 h)}{2 h} \\
& +\frac{1}{30} \frac{\eta(r+3 h)-\eta(r-3 h)}{2 h} \\
= & \frac{\left(45-9 \phi+\phi^{2}\right)}{30} \eta_{N=3}^{(1)}(r)+W^{\prime \prime}
\end{aligned}
$$

and

$$
\begin{aligned}
\eta_{N=9}^{(1)}(r)= & \frac{672}{420} \eta_{N=3}^{(1)}(r)-\frac{168}{420} \frac{\eta(r+2 h)-\eta(r-2 h)}{2 h} \\
& +\frac{32}{420} \frac{\eta(r+3 h)-\eta(r-3 h)}{2 h} \\
& -\frac{3}{420} \frac{\eta(r+4 h)-\eta(r-4 h)}{2 h} \\
= & \frac{\left(672-168 \phi+32 \phi^{2}-3 \phi^{3}\right)}{420} \eta_{N=3}^{(1)}(r)+W^{\prime \prime \prime},
\end{aligned}
$$

where

$$
\begin{aligned}
W^{\prime \prime}= & -\frac{9}{60} \frac{[w(r+2 h)-w(r-2 h)]}{h} \\
& +\frac{1}{60} \frac{[w(r+3 h)-w(r-3 h)]}{h} \\
= & \frac{\sqrt{164}}{160} \frac{W}{h} \approx 0.2134 \frac{W}{h},
\end{aligned}
$$

and

$$
\begin{aligned}
W^{\prime \prime \prime}= & -\frac{168}{840} \frac{[w(r+2 h)-w(r-2 h)]}{h} \\
& +\frac{32}{840} \frac{[w(r+3 h)-w(r-3 h)]}{h} \\
& -\frac{3}{840} \frac{[w(r+4 h)-w(r-4 h)]}{h} \\
= & \frac{\sqrt{58514}}{840} \frac{W}{h} \approx 0.2880 \frac{W}{h} .
\end{aligned}
$$


(a) 3 point

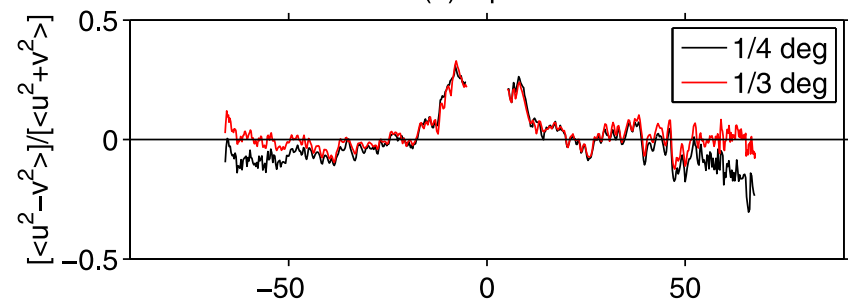

(b) 9 point

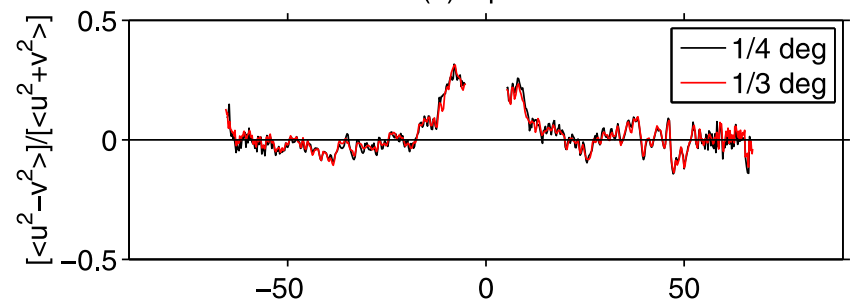

(c) 9 point with 7, 5, and 3 point fill-ins

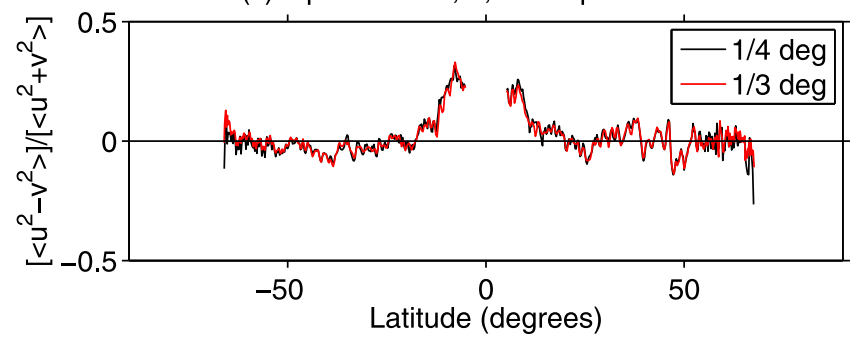

Figure 10. Normalized difference of time- and zonallyaveraged zonal and meridional velocity variance, $\left[<u^{2}-v^{2}>\right] /$ $\left[<u^{2}+v^{2}>\right]$, where [] is the zonal average operator and $<>$ is a time-averaging operator, computed from 16 years of AVISO products (December 30, 1992 through December $31,2008)$. Both the isotropic $1 / 3^{\circ}$ Mercator grid reference AVISO product and the anisotropic $1 / 4^{\circ}$ latitude-longitude grid reference AVISO product are used. Values of timeaveraged $u$ and $v$ are removed from the $u$ and $v$ fields before the difference is computed. Differences are computed using (a) 3-point stencils, (b) 9-point stencils, and (c) a blended product, in which first the 9-point stencil is used, then a 7-point stencil is used to fill in missing points along boundaries, followed by usage of a 5-point stencil, and finally a 3-point stencil.

[30] We are interested in the speed-dependent bias in the derivative estimate resulting from the 3 -point stencil relative to the 9-point stencil, so we write (31) in the form

$$
\eta_{N=3}^{(1)}(r)-\eta_{N=9}^{(1)}(r)=R B \eta_{N=9}^{(1)}(r)-\frac{1}{c} W^{\prime \prime \prime}
$$

where the relative bias $R B=\frac{1-c}{c}$ and $c$ is the coefficient in front of $\eta_{N=3}^{(1)}(r)$ in (31):

$$
c=\frac{672-168 \phi+32 \phi^{2}-3 \phi^{3}}{420} .
$$

The relative bias is a negative and increasing function on the interval $\phi \in[0,1)$. The bias with the smallest magnitude occurs in the limit of very strong autocorrelation $\phi \rightarrow 1$. In this limit, we have (ignoring the random parts)

$$
\begin{gathered}
\eta_{N=5}^{(1)}(r)=\frac{7}{6} \eta_{N=3}^{(1)}(r), \\
\eta_{N=7}^{(1)}(r)=\frac{37}{30} \eta_{N=3}^{(1)}(r), \\
\eta_{N=9}^{(1)}(r)=\frac{533}{420} \eta_{N=3}^{(1)}(r),
\end{gathered}
$$

which yields, for the differences between narrower stencil derivatives and the 9-point stencil derivative (again ignoring the random parts),

$$
\begin{aligned}
& \eta_{N=3}^{(1)}(r)-\eta_{N=9}^{(1)}(r)=-\frac{113}{420} \eta_{N=3}^{(1)}(r)=-\frac{113}{533} \eta_{N=9}^{(1)}(r), \\
& \eta_{N=5}^{(1)}(r)-\eta_{N=9}^{(1)}(r)=-\frac{43}{420} \eta_{N=3}^{(1)}(r)=-\frac{43}{533} \eta_{N=9}^{(1)}(r), \\
& \eta_{N=7}^{(1)}(r)-\eta_{N=9}^{(1)}(r)=-\frac{15}{420} \eta_{N=3}^{(1)}(r)=-\frac{15}{533} \eta_{N=9}^{(1)}(r) .
\end{aligned}
$$

[31] It is important to note that the random parts are correlated with the discrepancy between the 3-point and 9-point stencil estimate because it is the linear combination of the white-noise contribution $w$ to $\eta$ from points inside the 9-point stencil. Thus we do not expect the stochastic model to give quantitative predictions of the speed-dependent bias. Indeed, the stochastic model prediction that the 3-point stencil results will be biased with respect to the 9-point stencils by about $21 \%$, is an over-estimate. Least-squares fits to the plots in Figure 4 (based on altimetry data) yield speed-dependent biases in the range $4-7 \%$, and least squares fits to the plots in Figures $5 \mathrm{c}$ and $5 \mathrm{~d}$ (NLOM decimated by a factor of eight) yield speed-dependent biases in the range 7-10\%. The speeddependent bias indicated by the least squares fit to Figures $5 \mathrm{a}$ and $5 \mathrm{~b}$ (NLOM on its native high-resolution grid) is much smaller $(1-2 \%)$. Note that in this regard the appearance of the plots in Figure 5 is deceptive. There are many more points plotted in Figures $5 \mathrm{a}$ and $5 \mathrm{~b}$ than in Figures $5 \mathrm{c}$ and $5 \mathrm{~d}$. Hence there are a greater number of points with large misfits in Figures $5 \mathrm{a}$ and $5 \mathrm{~b}$; however the percentage of points having a large misfit is much smaller. The smaller bias on the higher resolution grid is consistent with the discussion of the von Neumann analysis in section 3. Clearly, in all of the plots in Figures 4 and 5, discrepancies between narrow stencil estimates and 9-point stencil estimates at individual grid points can be much larger than the least-squares slopes quoted above. The stochastic model appears to qualitatively explain the speed-dependent bias seen earlier in the paper, though the quantitative estimate of the bias is too large. The stochastic model indicates that this speed-dependent bias will be seen even if derivatives are taken at very small grid spacing. This explains why a speed-dependent bias was seen even on the native high-resolution NLOM grid.

\section{Effect of Stencil Width on Estimates of Anisotropy in Kinetic Energy}

[32] One of our motivations for investigating the effects of stencil width on velocity estimation is to determine whether 
Table 2. Formulae for Noise-Suppressing Differentiators ${ }^{\mathrm{a}}$

\begin{tabular}{|c|c|}
\hline$N^{\mathrm{b}}$ & Formulae \\
\hline \multicolumn{2}{|r|}{ Low-Noise Lanczos Differentiator ${ }^{\mathrm{c}}$} \\
\hline 5 & $\frac{2 \eta(r+2 h)+\eta(r+h)-\eta(r-h)-2 \eta(r-2 h)}{10 h}$ \\
\hline 7 & $\frac{3 \eta(r+3 h)+2 \eta(r+2 h)+\eta(r+h)-\eta(r-h)-2 \eta(r-2 h)-3 \eta(r-3 h)}{28 h}$ \\
\hline 9 & $\frac{4 \eta(r+4 h)+3 \eta(r+3 h)+2 \eta(r+2 h)+\eta(r+h)-\eta(r-h)-2 \eta(r-2 h)-3 \eta(r-3 h)-4 \eta(r-4 h)}{60 h}$ \\
\hline & Super Lanczos Low-Noise Differentiator ${ }^{\mathrm{c}}$ \\
\hline 7 & $\frac{-22 \eta(r+3 h)+67 \eta(r+2 h)+58 \eta(r+h)-58 \eta(r-h)-67 \eta(r-2 h)+22 \eta(r-3 h)}{252 h}$ \\
\hline 9 & $\frac{-86 \eta(r+4 h)+142 \eta(r+3 h)+193 \eta(r+2 h)+126 \eta(r+h)-126 \eta(r-h)-193 \eta(r-2 h)-142 \eta(r-3 h)+86 \eta(r-4 h)}{1188 h}$ \\
\hline 5 & $\begin{array}{l}\text { Smooth Noise-Robust }(n=2) \text { Differentiator } \\
\qquad \underline{\eta(r+2 h)+2 \eta(r+h)-2 \eta(r-h)-\eta(r-2 h)}\end{array}$ \\
\hline 5 & $\begin{array}{c}8 h \\
\eta(r+3 h)+4 \eta(r+2 h)+5 \eta(r+h)-5 \eta(r-h)-4 \eta(r-2 h)-\eta(r-3 h)\end{array}$ \\
\hline 7 & $32 h$ \\
\hline 9 & $\frac{\eta(r+4 h)+6 \eta(r+3 h)+14 \eta(r+2 h)+14 \eta(r+h)-14 \eta(r-h)-14 \eta(r-2 h)-6 \eta(r-3 h)-\eta(r-4 h)}{128 h}$ \\
\hline & Smooth Noise-Robust $(n=4)$ Differentiator ${ }^{\mathrm{c}}$ \\
\hline 7 & $\frac{-5 \eta(r+3 h)+12 \eta(r+2 h)+39 \eta(r+h)-39 \eta(r-h)-12 \eta(r-2 h)+5 \eta(r-3 h)}{96 h}$ \\
\hline 9 & $\frac{-2 \eta(r+4 h)-\eta(r+3 h)+16 \eta(r+2 h)+27 \eta(r+h)-27 \eta(r-h)-16 \eta(r-2 h)+\eta(r-3 h)+2 \eta(r-4 h)}{96 h}$ \\
\hline
\end{tabular}

this had any impact on the estimation of the anisotropy of time- and zonally-averaged surface ocean velocity variance fields by Scott et al. [2008]. In this section especially it is of interest to examine results computed from both anisotropically and isotropically gridded altimeter products. In this as in earlier sections we continue to focus on derivative estimates made with classic centered differences. As in the work of Scott et al. [2008] (see their Figure 9b), we compute the quantity

$$
M_{z 2}=\frac{\left.\left[<u^{2}-v^{2}\right\rangle\right]}{\left.\left[<u^{2}+v^{2}\right\rangle\right]},
$$

where $<>$ is a time-averaging operator. See also Ducet et al. [2000] and Scharffenberg and Stammer [2010], who performed similar computations on altimeter data. As in the work of Scott et al. [2008], for this calculation we remove time averages from $u$ and $v$ before computing $M_{z 2}$. Here we compute $M_{z 2}$ over 16 years of satellite altimetry data, from December 30, 1992 through December 31, 2008. Figure 10a reveals that 3-point stencil computations of $M_{z 2}$ yield different results in mid-to-high latitudes on the $1 / 4^{\circ}$ grid than on the $1 / 3^{\circ}$ grid, despite the fact that the $1 / 4^{\circ}$ grid AVISO product is derived from the $1 / 3^{\circ}$ product by simple bi-linear interpolation. The discrepancy between results on the two different grids is expected based on the discussion in the previous sections. On the $1 / 4^{\circ}$ grid, the bias toward smaller values of kinetic energy in the 3-point stencil computation is especially pronounced for $u$ at mid-to-high latitudes (compare Figure $6 \mathrm{c}$ to Figures $6 \mathrm{a}, 6 \mathrm{~b}$, and $6 \mathrm{~d}$ ). At these latitudes, the AVISO regridding from the original Mercator $1 / 3^{\circ}$ isotropic grid to the $1 / 4^{\circ}$ anisotropic grid apparently degrades $u$ more than $v$. Again, this is because $u$ is based on derivatives in $y$, and at high latitudes the $y$ spacing is coarser on the $1 / 4^{\circ}$ anisotropic grid than on the Mercator $1 / 3^{\circ}$ isotropic grid.
Since the $x$-spacing is always less on the $1 / 4^{\circ}$ anisotropic grid than on the Mercator $1 / 3^{\circ}$ isotropic grid, $v$ does not suffer from this degradation.

[33] Consistent with earlier discussions, Figure 10b demonstrates that $M_{z 2}$ computed with 9-point stencils yields very similar results when computed on the $1 / 4^{\circ}$ grid as on the $1 / 3^{\circ}$ grid. However, a disadvantage of utilizing wider stencils is that more information is lost along the boundaries where a full span of the stencil does not exist because of land. To alleviate this problem, we have also created a blended velocity estimate, which begins with velocities computed using 9-point stencils, then where possible fills in missing velocities computed using 7-point stencils, followed by those computed from 5-point stencils, and finally by those computed from 3-point stencils. This blended estimate has the disadvantage that the "quality" of derivatives is not uniform across all grid points, and the advantage that the number of grid points for which a velocity estimate is available is as large as in the 3-point estimates. In Figure 10c we show $M_{z 2}$ computed from the blended estimate. As in Figure 10b, the differences between $M_{z 2}$ values computed on the $1 / 3^{\circ}$ versus $1 / 4^{\circ}$ AVISO grids are much smaller than seen in Figure $10 \mathrm{a}$ (3-point stencil computation). Discrepancies between $1 / 3^{\circ}$ and $1 / 4^{\circ}$ results, and between pure 9 -point stencil results and results from the blended product, are largest at high southern and northern latitudes.

[34] Scott et al. [2008] and Scharffenberg and Stammer [2010] both found near isotropy in the mid- and highlatitude zonally averaged values of the difference between zonal and meridional velocity variances. Scott et al. [2008] utilized the Mercator $1 / 3^{\circ}$ isotropic grid AVISO product while Scharffenberg and Stammer [2010] utilized data from the tandem phase of the JASON/TOPEX missions. Figure 10 shows that near-isotropy (small values of $M_{z 2}$ ) is also seen when wider stencils are used. We thus conclude that the 

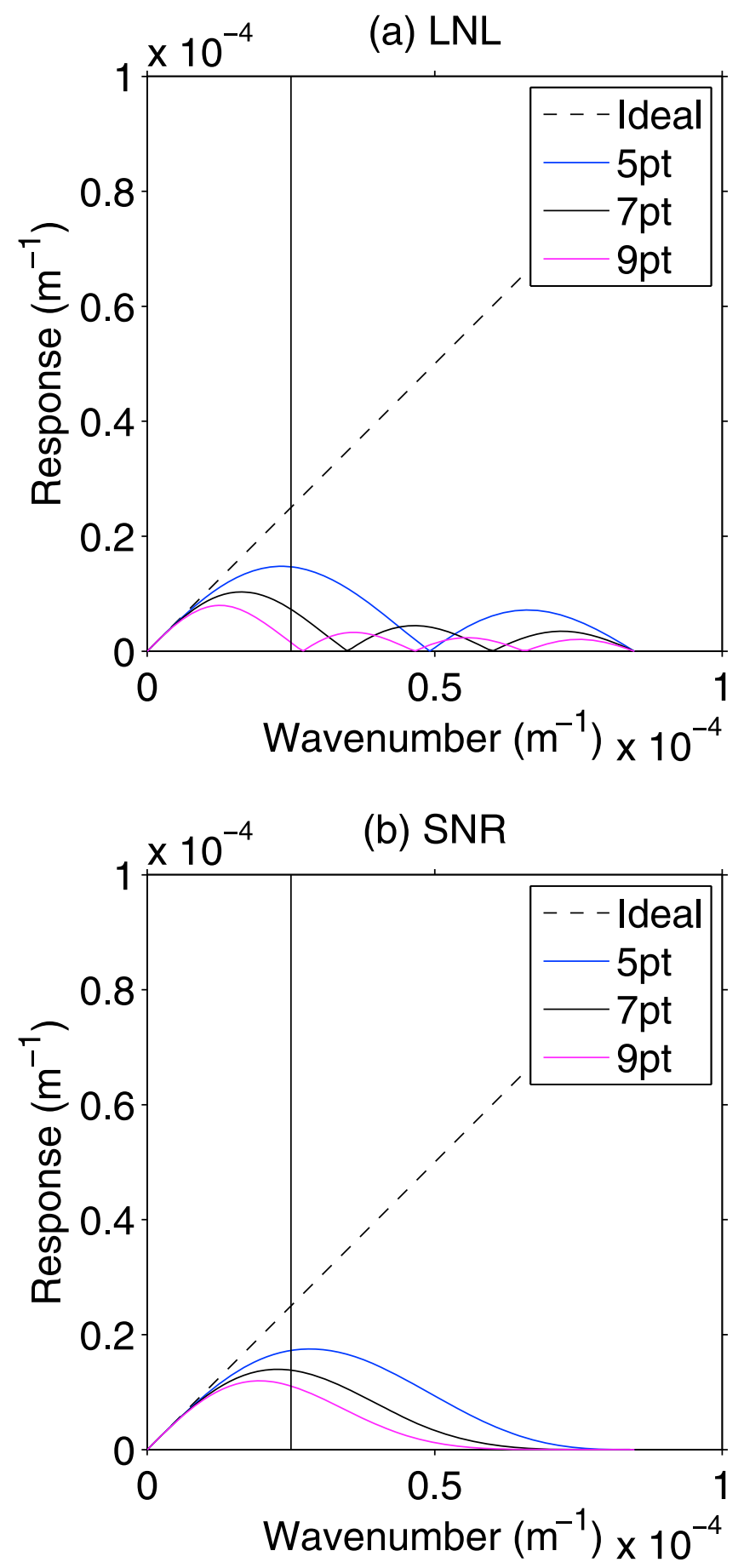

Figure 11. Response of an ideal differentiator and of 5-, 7-, and 9-point stencils, as a function of wave number, on $1 / 3^{\circ}$ AVISO grid, for (a) Low-noise Lanczos (LNL) differentiator and (b) smooth noise-robust (SNR; $n=2$ ) differentiator. The grid spacings used are $37 \mathrm{~km}$, and the response is the absolute value $|\Lambda|$ of the eigenvalue of the von Neumann analysis. See text for more details. Vertical lines are drawn at the wave number $(40 \mathrm{~km})^{-1}$, corresponding approximately to the $2-3^{\circ}$ wavelength resolution limitation of the AVISO sea surface height fields. inference of Scott et al. [2008] and Scharffenberg and Stammer [2010], that the zonally averaged oceanic kinetic energy is nearly isotropic at midlatitudes, is not an artifact of the three-point stencil used to compute derivatives in these studies.

\section{Effects of Noise}

[35] As stated by Holoborodko (2011), in many applications, ideal differentiators, and classic centered differences which closely approximate ideal differentiators over a wide range of scales, are problematic. This is because in many applications there is significant noise at high frequencies (or wave numbers), and the high frequency noise should be suppressed if one wants reasonable estimates of the derivative at low frequencies. A common procedure for doing this is to first smooth the data with a least-squares polynomial fit and to then estimate the derivative using this polynomial. An extensive literature on smoothing and differentiation through least-squares fitting exists in the chemical and signal processing community [Savitzky and Golay, 1964; Steiner et al., 1972; Gorry, 1990; Luo et al., 2005]. We found the discussion of Holoborodko (2011) especially useful.

[36] Following the discussion of Holoborodko (2011), we considered a particular class of Savitzky-Golay filters, known as "low-noise Lanczos" and "super Lanczos lownoise" differentiators, as well as a class of filter designed by Holoborodko (2011) and known as a "smooth noise-robust" differentiator. Table 2 lists the first-derivative formulae for some of these filters. As a check, we derived these formulae for ourselves, and obtained the same answers as Holoborodko (2011).

[37] In Figure 11, we display results of the von Neumann analysis for the low-noise Lanczos and smooth noise-robust $(n=2)$ differentiators. See Holoborodko (2011) for a full exposition and derivation, which includes an explanation of the meaning of $n$ in the smooth noise-robust differentiators. As shown in the figure, and as described by Holoborodko (2011), these differentiators are designed to suppress noise at high wave numbers, while remaining close to the idealdifferentiator behavior at low wave numbers. However, a problem for these low-noise differentiators is that they deviate significantly from the ideal differentiator response over about half of the range of wave numbers lower than the $2-3^{\circ}$ wavelength resolution limitation of the AVISO sea surface height fields. Thus, these low-noise differentiators suppress much of the signal as well as the noise. The spectra shown in Figure 1 demonstrate that there is little variance in the AVISO data in wave numbers higher than those representing the resolution limitation. Thus, for the applications relevant to this paper, noise suppression at high wave numbers may be less important than retaining near-ideal differentiation at low wave numbers.

[38] To test this further, we looked at differences between 5-point, 7-point, and 9-point stencil results with these lownoise differentiators applied to a snapshot of the $1 / 3^{\circ}$ AVISO data set (Figure 12). The discrepancies between derivatives computed with narrow and wide stencils is much greater using these low-noise differentiators than when using classic centered differences. We obtained similar, though somewhat less extreme, results using the "super Lanczos lownoise" differentiators and the "smooth-noise robust $(n=4)$ " 
(a) Centered differences

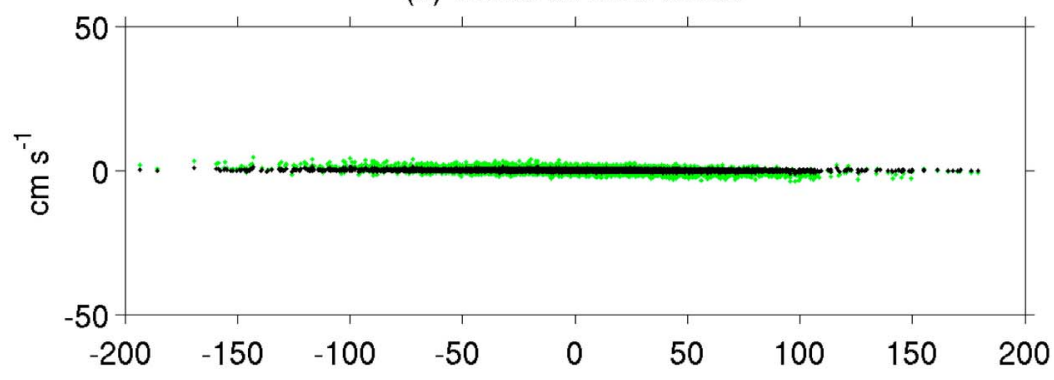

(b) Low-noise Lanczos

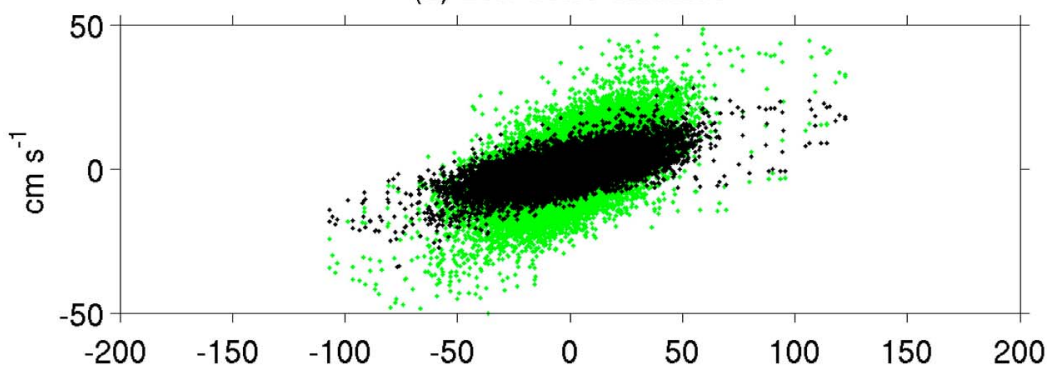

(c) Smooth noise-robust

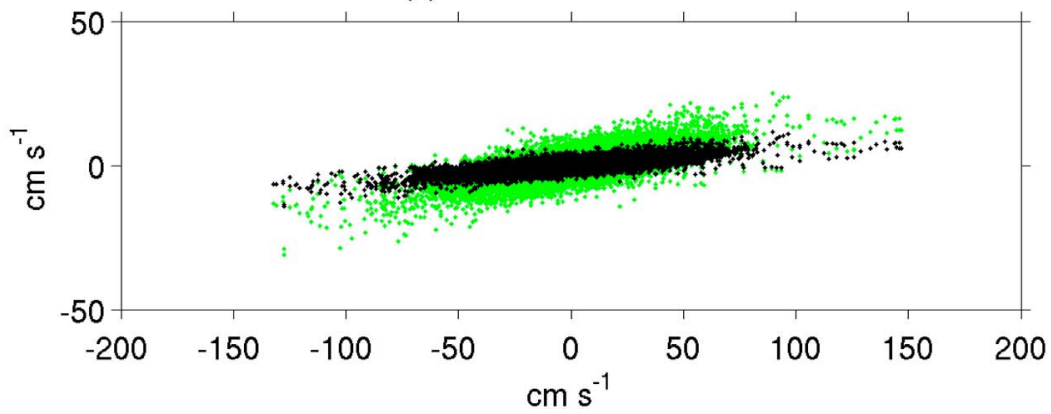

Figure 12. Difference between zonal velocity $u$ computed with 5-point and 9-point stencils (green dots), and with 7-point and 9-point stencil results (black dots), for (a) classic centered difference formulae used elsewhere in the text, (b) low-noise Lanczos differentiators, and (c) smooth noise-robust $(n=2)$ differentiators. Differences are computed for the November 5, 2008 snapshot of the $1 / 3^{\circ}$ AVISO data set.

differentiators (results not shown). If we accept "convergence" with increasing stencil width as an important criterion, we conclude that the low-noise differentiators are not as well-suited as classic centered differences for computing derivatives on current-generation altimeter data. For next generation wide-swath satellite altimeter data $[F u$ and Ferrari, 2008], the noise characteristics of the signals are as yet unknown. Although the instrument is expected to perform some amount of averaging over the raw measurements, the amount of smoothing will be much less than that used in the construction of current-generation gridded satellite altimeter data. As a result, noise-suppressing differentiators are likely to become more necessary and important with the coming of high resolution wide-swath data.

[39] We also investigated the extent to which derivative estimates made with classic centered differences are affected by noise in the altimeter data. For this purpose we have constructed three types of random noise fields on the $1 / 3^{\circ}$ AVISO grid. The first is simply a noise field which is random from one point to another. The second and third versions are produced from Blackman filtering applied to the random noise. The second version utilizes a Blackman filter which goes to zero at a distance of $2^{\circ}$, while the third goes to zero at $3^{\circ}$. Each of the three fields is multiplied by constants, such that we end up with fields having RMS values of $0.1,0.5$, and $1 \mathrm{~cm}$. This yields 9 random noise fields in all. In Figure 13 we display differences in zonal velocity estimated with 9 point stencils, with and without the RMS $1 \mathrm{~cm}$ amplitude, $3^{\circ}$ filtered noise. We compare these differences (black dots) to the differences between zonal velocity estimated using 3 point versus 9 point stencils (red dots, no extra noise included). The noise adds an uncertainty in the velocity estimates which is comparable in magnitude to the differences one sees in estimates made with narrower stencils. Obviously, the size of the noise-induced error decreases with decreasing RMS amplitude. The error increases with decreasing horizontal scale; the error with $2^{\circ}$ noise is larger than with $3^{\circ}$ noise, and the error with random spatial noise is larger still (results not shown). However, in contrast to the speed-dependent bias one sees with narrower stencils, the uncertainty added by 


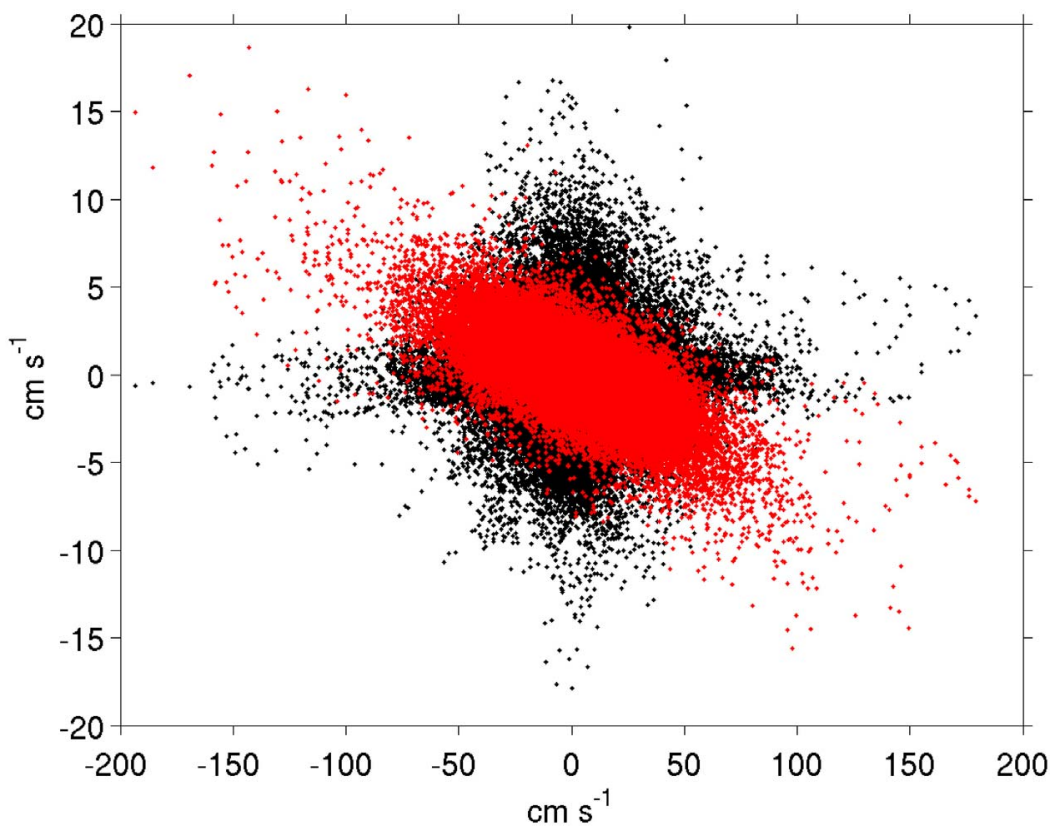

Figure 13. Difference between zonal velocity $u$ computed from the November 5, 2008 snapshot of the $1 / 3^{\circ}$ AVISO data set with 3-point minus 9-point stencils (red dots), and between $u$ computed with 9-point stencils with $1 \mathrm{~cm}$ RMS random noise filtered to $3^{\circ}$ added to AVISO, minus $u$ computed without the added noise (black dots).

these noise fields is not biased in an obvious way. Instead it appears to be distributed evenly around zero, with larger excursions occurring at smaller velocities. In addition, the effects of the noise are just as large with 3-point stencils as with 9-point stencils (not shown). Therefore the wider stencils are not a disadvantage in this exercise. The differences in estimates of the derivatives of the noise fields decrease with increasing stencil width, just as differences in the derivative estimates of the AVISO signals do (not shown).

\section{Summary and Discussion}

[40] Drawing on the well-developed numerical methods literature, we have shown that classic centered-difference estimates of geostrophic velocities and vorticities from gridded products of satellite altimeter-derived sea surface height lie closer to results obtained with an "ideal differentiator" as stencil width increases. This is especially true for mid-to-high latitude velocities computed on the $1 / 4^{\circ}$ AVISO grid, which is anisotropic (has different grid spacings in the east-west versus north-south directions). Similar computations using NLOM, a high-resolution numerical model, demonstrate that the impact of stencil width on geostrophic velocity estimation is not an artifact of the particular procedures and errors involved in creating gridded satellite altimeter products. A stochastic model developed here qualitatively explains the speed-dependent biases seen in velocity estimates made with narrow (3-point) stencils.

[41] This study was inspired in part by our earlier study of the anisotropy of ocean surface velocity variances [Scott et al., 2008], which utilized the traditional thin (3-point) stencils on the $1 / 3^{\circ}$ Mercator AVISO grid. We have shown here that usage of the 3-point stencils which are widely used in the oceanographic literature gives incorrect estimates of the anisotropy of velocity variance on the $1 / 4^{\circ}$ anisotropic AVISO grid; we recommend the use of 7-point stencils instead. Although the analyses in sections 2-7 show clearly that applications that require precise geostrophic velocity estimates should utilize wider stencils, the results of section 8 show that the general conclusions of Scott et al. [2008] and Scharffenberg and Stammer [2010] that zonally averaged velocity variance in midlatitudes is nearly isotropic still pertain when the derivatives are computed more accurately with wider stencils. Our study also has relevance to the proposed future wide-swath satellite altimeter mission [Fu and Ferrari, 2008], which is expected to map sea surface heights at resolutions about 20-30 times higher than the AVISO sea surface height fields. Consistent with predictions from our stochastic model, our analysis of high resolution NLOM output indicates that even at high horizontal resolution (small grid spacing) there are significant differences at individual grid points between velocities computed via 3 -point stencils and wider stencils. Thus wider stencils should be of interest for future satellite altimeter missions as well as for present ones. In future altimeter missions, the data may begin to approach what is the norm in many other fields, that is, a well resolved low-wavelength signal with significant noise at higher wavelengths. In this case, noisesuppressing differentiators such as those used extensively in the chemical and signal processing communities will become of greater interest to oceanographers.

[42] Acknowledgments. The authors thank three anonymous reviewers whose comments led to the inclusion of section 9 and to many other improvements in the manuscript. B.K.A. acknowledges helpful conversations with David Amundsen, Patrick Cummins, Sarah Gille, and Alfredo Wetzel, and funding provided by Office of Naval Research grant N00014-11-1-0487 and National Science Foundation (NSF) grants OCE0924481 and OCE-09607820. R.B.S. acknowledges funding provided by 
NSF grants OCE-0960834 and OCE-0851457, a contract with the National Oceanography Centre, Southampton, and a NASA subcontract to Boston University. D.B.C.'s contributions to this study were supported by NASA grant NNX08AR37G. J.F.S. and J.G.R. were supported by the projects "Global and remote littoral forcing in global ocean models" and "Ageostrophic vorticity dynamics of the ocean", respectively, both sponsored by the Office of Naval Research under program element 601153N. This is NRL contribution NRL/JA/7320-2010-557 and has been approved for public release. This is UTIG contribution 2397.

\section{References}

Arbic, B. K., and G. R. Flierl (2004), Baroclinically unstable geostrophic turbulence in the limits of strong and weak bottom Ekman friction: Application to mid-ocean eddies, J. Phys. Oceanogr., 34, 2257-2273.

Chelton, D. B., M. G. Schlax, R. M. Samelson, and R. A. de Szoeke (2007), Global observations of large oceanic eddies, Geophys. Res. Lett., 34, L15606, doi:10.1029/2007GL030812.

Chelton, D. B., M. G. Schlax, and R. M. Samelson (2011), Global observations of nonlinear mesoscale eddies, Prog. Oceanogr., 91, 167-216.

Cushman-Roisin, B., and J.-M. Beckers (2010), Introduction to Geophysical Fluid Dynamics: Physical and Numerical Aspects, 875 pp., Academic, San Diego, Calif.

Ducet, N., P.-Y. Le Traon, and G. Reverdin (2000), Global high-resolution mapping of ocean circulation from TOPEX/Poseidon and ERS-1 and -2, J. Geophys. Res., 105, 19,477-19,498.

Fu, L.-L., and A. Cazenave (Eds.) (2001), Satellite Altimetry and Earth Sciences: A Handbook of Techniques and Applications, 463 pp. Academic, San Diego, Calif.

Fu, L.-L., and R. Ferrari (2008), Observing oceanic submesoscale processes from space, Eos Trans. $A G U, 89(48), 488$.

Gorry, P. A. (1990), General least-squares smoothing and differentiation by the convolution (Savitzky-Golay) method, Anal. Chem., 62, 570-573.

Hurlburt, H. E., and J. D. Thompson (1980), A numerical study of Loop Current intrusions and eddy shedding, J. Phys. Oceanogr., 10, $1611-1651$

Le Traon, P.-Y., F. Nadal, and N. Ducet (1998), An improved mapping method of multi-satellite altimeter data, J. Atmos. Oceanic Technol., 15 , 522-534.

LeVeque, R. J. (2007), Finite Difference Methods for Ordinary and Partial Differential Equations: Steady-State and Time-Dependent Problems, 341 pp., Soc. for Ind. and Appl. Math., Philadelphia, Pa.

Luo, J., K. Ying, P. He, and J. Bai (2005), Properties of Savitzky-Golay digital differentiators, Digital Signal Process., 15, 122-136.

Mathews, J. H., and K. D. Fink (2004), Numerical Methods: Using Matlab, 4th ed., 680 pp., Prentice Hall, Upper Saddle River, N. J.

Pedlosky, J. (1987), Geophysical Fluid Dynamics, 2nd ed., 710 pp., Springer, New York.
Savitzky, A., and M. J. E. Golay (1964), Smoothing and differentiation of data by simplified least squares procedures, Anal. Chem., 36, 1627-1639.

Scharffenberg, M. G., and D. Stammer (2010), Seasonal variations of the large-scale geostrophic flow field and eddy kinetic energy inferred from the TOPEX/Poseidon and Jason-1 tandem mission data, J. Geophys. Res., 115, C02008, doi:10.1029/2008JC005242.

Scott, R. B., and F. Wang (2005), Direct evidence of an oceanic inverse kinetic energy cascade from satellite altimetry, J. Phys. Oceanogr., 35, 1650-1666.

Scott, R. B., B. K. Arbic, C. L. Holland, A. Sen, and B. Qiu (2008), Zonal versus meridional velocity variance in satellite observations and realistic and idealized ocean circulation models. Ocean Modell., 23, 102-112, doi:10.1016/j.ocemod.2008.04.009.

Scott, R. B., et al. (2010), Integrating satellite altimetry and key observations: What we've learned, and what's possible with new technologies, in Proceedings of the "OceanObs'09: Sustained Ocean Observations and Information for Society" Conference, 21-25 September 2009, Venice, Italy, ESA Publ., WPP-366, Eur. Space Agency, Paris.

Shriver, J. F., H. E. Hurlburt, O. M. Smedstad, A. J. Wallcraft, and R. C. Rhodes (2007), $1 / 32^{\circ}$ real-time global ocean prediction and value-added over $1 / 16^{\circ}$ resolution, J. Mar. Syst., 65, 3-26.

Stammer, D. (1997), Global characteristics of ocean variability estimated from regional TOPEX/Poseidon altimeter measurements, J. Phys. Oceanogr., 27, 1743-1769.

Steiner, J., Y. Termonia, and J. Deltour (1972), Comments on smoothing and differentiation of data by simplified least square procedure, Anal. Chem., 44, 1906-1909.

Strikwerda, J. C. (2004), Finite Difference Schemes and Partial Differential Equations, 2nd ed., 435 pp., Soc. for Ind. and Appl. Math., Philadelphia, Pa.

Vallis, G. K. (2006), Atmospheric and Oceanic Fluid Dynamics, 745 pp., Cambridge Univ. Press, New York.

Wallcraft, A. J., A. B. Kara, H. E. Hurlburt, and P. A. Rochford (2003), The NRL Layered Ocean Model (NLOM) with an embedded mixed layer sub-model: Formulation and tuning, J. Atmos. Oceanic Technol., 20, 1601-1615.

$\mathrm{Xu}, \mathrm{Y}$., and L.-L. Fu (2011), Global variability of the wavenumber spectrum of oceanic mesoscale turbulence, J. Phys. Oceanogr., 41, 802-809.

B. K. Arbic, Department of Earth and Environmental Sciences, University of Michigan, 2534 C. C. Little Bldg., Ann Arbor, MI 481091005, USA. (arbic@umich.edu)

D. B. Chelton, College of Oceanic and Atmospheric Sciences, Oregon State University, Corvallis, OR 97331, USA.

J. G. Richman and J. F. Shriver, Oceanography Division, Naval Research Laboratory, Stennis Space Center, MS 39529, USA.

R. B. Scott, Institute for Geophysics, Jackson School of Geosciences, University of Texas at Austin, Austin, TX 78758, USA. 